\title{
STATICALLY TAME PERIODIC HOMEOMORPHISMS OF COMPACT CONNECTED 3-MANIFOLDS. I. HOMEOMORPHISMS CONJUGATE TO ROTATIONS OF THE 3-SPHERE ${ }^{1}$
}

BY

EDWIN E. MOISE

To David Beres

\begin{abstract}
Let $f$ be a homeomorphism of the 3-sphere onto itself, of finite period $n$, and preserving orientation. Suppose that the fixed-point set $F$ of $f$ is a tame 1-sphere. It is shown that (1) the 3-sphere has a triangulation $K\left(\mathbf{S}^{3}\right)$ such that $F$ forms a subcomplex of $K\left(\mathbf{S}^{3}\right)$ and $f$ is simplicial relative to $K\left(\mathbf{S}^{3}\right)$. Suppose also that $F$ is unknotted. It then follows that (2) $f$ is conjugate to a rotation.
\end{abstract}

1. Statement of results. The following question has been proposed by R. H. Bing [ $\mathbf{B}_{1}$ ]. Let $f: \mathbf{R}^{3} \leftrightarrow \mathbf{R}^{3}$ be a periodic homeomorphism of Cartesian 3-space onto itself, with a straight line as its fixed-point set. Does it follow that $f$ is conjugate to a rotation? We shall show that this conclusion does follow, if we assume also that $f$ preserves orientation, or that the period of $f$ is a prime. See Theorems 1.5 and 1.6 below. In the sequel to the present paper, we shall show that Bing's conjecture is true in general.

By an $n$-manifold we mean a separable metric space $M$ in which each point has a neighborhood homeomorphic to Cartesian $n$-space $\mathbf{R}^{n}$. Thus $M$ is not necessarily compact or connected. If $M$ is separable and metric, and each point has a neighborhood which is an $n$-cell, then $M$ is an $n$-manifold with boundary. Int $M$ is the set of all points of $M$ that have open Cartesian neighborhoods, and $\mathrm{Bd} M$ is the set of all points of $M$ that do not.

Let $M$ be a 3-manifold, and let $A$ be a subset of $M$. Suppose that there is a triangulation $K$ of $M$, and a homeomorphism $h: M \leftrightarrow M$, such that $h(A)$ is a polyhedron relative to $K$. Then $A$ is tame. (For a general definition of a polyhedron, see the discussion just before Theorem 3.3 below.) By the Hauptvermutung $\left[\mathbf{M}_{\mathbf{5}}\right.$ ], this condition is independent of the choice of $K$, and so the above definition of tameness agrees with the classical definition, due to

Received by the editors September 25, 1974 and, in revised form, November 10, 1976 and October 3, 1977 on the author's initiative.

AMS (MOS) subject classifications (1970). Primary 57A10; Secondary 57E10, 57E25.

Key words and phrases. Periodic homeomorphism, 3-sphere, fixed-point set.

'The research reported here was supported by the National Science Foundation, under grants GP 29265 and MCS-76-07282. 
Artin and Fox [FA], for the cases in which $M$ is $\mathbf{R}^{3}$ or the 3-sphere $\mathbf{S}^{3}$.

THEOREM 1.1. Let $M$ be a connected 3-manifold which is a subspace of $\mathbf{S}^{3}$. Let $f: M \leftrightarrow M$ be a homeomorphism of period $n$, preserving orientation and with fixed-point set $F$. Suppose that $F$ is a 1-sphere which is the boundary of a tame 2-cell $D \subset \mathbf{S}^{3}$, and that $f$ has period exactly $n$ at each point of $M-F$. Let $\operatorname{Pr}$ : $M \rightarrow \Omega$ be the projection of $M$ onto the orbit space $\Omega$ of $f$. Then $\Omega$ is a 3-manifold, and $\operatorname{Pr} F$ is tame in $\Omega$.

This is Theorem 11.8 below.

Let $f: M \leftrightarrow M$ be a periodic homeomorphism of a 3-manifold $M$ onto itself. If for each $i$, the fixed-point set $F_{i}$ of $f^{i}$ is tame, then $f$ is statically tame. (Thus the $f$ of Theorem 1.1 is statically tame, since for each $i>0$ we have $F_{i}=F$.) If $M$ has a triangulation $K(M)$ relative to which $f$ is simplicial, then $f$ is tame. In the sequel to the present paper, it will be shown that if $M$ is compact and connected, and $f$ is statically tame, then $f$ is tame. Theorem 1.1 is, in effect, a special case of this proposition, as the following theorem shows.

THEOREM 1.2. Under the conditions of Theorem 1.1, $M$ has a triangulation $K(M)$ such that $F$ forms a subcomplex of $K(M)$ and $f$ is simplicial relative to $K(M)$.

This is a consequence of Theorem 1.1. Let $\tilde{U}=M-F$, and let $\rho=\operatorname{Pr} \mid \tilde{U}$ : $\tilde{U} \rightarrow U \subset \Omega$, so that $\rho$ is an $n$-sheeted covering. We know that $\operatorname{Pr} F$ is tame in $\Omega$. Let $K(\Omega)$ be a triangulation of $\Omega$ in which $\operatorname{Pr} F$ forms a subcomplex, such that if $\sigma \in K(\Omega)$, and $\sigma \cap \operatorname{Pr} F \neq \varnothing$, then $\sigma \cap \operatorname{Pr} F$ is a face of $\sigma$. Let $K(M)$ be the lifting of $K(\Omega)$ to $M$. (To be exact, the simplices of $K(M)$ are the sets $\operatorname{Pr}^{-1} \sigma(\sigma \in K(\Omega), \sigma \subset F)$ and the closures of the components of the nonempty sets $\operatorname{Pr}^{-1}(\sigma-\operatorname{Pr} F)(\sigma \in K(\Omega))$.) Then $f$ is simplicial relative to $K(M)$.

THEOREM 1.3. Let $f$ be a periodic homeomorphism $\mathbf{S}^{3} \leftrightarrow \mathbf{S}^{3}$, of period $n$, preserving orientation, and suppose that the fixed-point set $F$ of $f$ is a 1-sphere. Then $f$ has period exactly $=n$ at each point of $\mathbf{S}^{3}-F$.

The proof is in $\S 2$.

A tame 1-sphere in a 3-manifold $M$ is unknotted if it is the boundary of a tame 2-cell.

THEOREM 1.4. Let $K(M)$ be a triangulation of a 3-manifold $M$, and let $F$ be an unknotted 1-sphere which is polyhedral relative to $K(M)$. Then $F$ is the boundary of a 2-cell $D$ which is polyhedral relative to $M$.

The proof is in $\$ 12$.

It was shown in [M] that if $f: \mathbf{S}^{3} \leftrightarrow \mathbf{S}^{3}$ is a homeomorphism of period $n$, preserving orientation, and simplicial relative to some triangulation of $\mathbf{S}^{3}$, 
with an unknotted polygon as its fixed-point set, then $f$ is conjugate to a rotation. (For a simpler proof, see $\mathbf{P}$. A. Smith $\left[\mathbf{S}_{2}\right]$.) Thus the preceding three theorems fit together to give the following.

THEOREM 1.5. Let $f$ be a periodic homeomorphism $\mathbf{S}^{3} \leftrightarrow \mathbf{S}^{3}$, preserving orientation, and suppose that the fixed-point set of $f$ is a tame unknotted 1-sphere. Then $f$ is conjugate to a rotation.

Obviously this is a restatement of Bing's conjecture, for the case in which orientation is preserved.

THEOREM 1.6. Let $f$ be a homeomorphism $\mathbf{S}^{3} \leftrightarrow \mathbf{S}^{3}$, of prime period $p$, and suppose that the fixed-point set of $f$ is a tame unknotted 1-sphere. Then $f$ is conjugate to a rotation.

To reduce this to Theorem 1.5, we need to show that the $f$ of Theorem 1.6 preserves orientation. If $p$ is odd, this is clear: if $f$ reversed orientation, then every odd power of $f$ would reverse orientation, which is impossible, because $f^{p}$ is the identity. We suppose, then, that $p=2$. It has been shown by Glen $\mathrm{E}$. Bredon that if $h: N \leftrightarrow N$ is an involution of a compact connected $n$-manifold, and $H$ is a component of the fixed-point set of $h$, then $n-\operatorname{dim} H$ is even if and only if $G$ preserves local orientation at some point of $H$. (See Borel [ $\mathbf{B}_{4}, \mathrm{p}$. 79] or Bredon $\left[\mathbf{B}_{5}\right]$.) In the present case, $n-\operatorname{dim} H=3-1$. Since $f$ preserves local orientation at some point, $f$ preserves orientation, as desired.

2. Proof of Theorem 1.3. A classical result of P. A. Smith ( $\left[S_{1}\right.$, p. 708]) asserts that if $h: \mathbf{S}^{3} \leftrightarrow \mathbf{S}^{3}$ preserves orientation, has finite period (either prime or composite), and has a fixed point, then the fixed-point set $H$ of $h$ is a 1 -sphere. Given $f$ and $n$ as in Theorem 1.3, suppose that $f$ has period $k<n$ at some point of $\mathbf{S}^{3}-F$. Then $k>1, k$ divides $n$, and $n=k m$, where $1<m<$ $n$. Let $g=f^{k}$, and let $G$ be the fixed-point set of $g$. Then $g$ has period $m$, and $F$ is a proper subset of $G$. This is impossible, because $F$ and $G$ are 1-spheres.

3. PL approximations and the Loop Theorem. Here we state various known results, some of them in modified forms.

THEOREM 3.1. Every 3-manifold is triangulable. So also is every 3-manifold with boundary.

(See Theorem 2 of $\left[\mathbf{M}_{\mathbf{5}}\right]$ and Theorem 9.1 of $\left[\mathbf{M}_{\mathbf{8}}\right]$. See also Bing $\left[\mathbf{B}_{2}\right]$.)

For each complex $K,|K|$ denotes the associated polyhedron. We shall need to observe the distinction between these, because often we shall be dealing with different triangulations $K_{1}(S), K_{2}(S)$ of the same space $S$, in cases where 
there is no PL relation between $K_{1}(S)$ and $K_{2}(S)$. If $|K|$ is a manifold (or a manifold with boundary), then $K$ will be called a PL manifold (or a PL manifold with boundary).

Let $U$ be a topological space, and let $\phi$ be a function $U \rightarrow \mathbf{R}$, where $\mathbf{R}$ is the set of all real numbers. (Here $\phi$ is not necessarily continuous.) Suppose that for each compact subset $A$ of $U$ there is an $\varepsilon_{A}>0$ such that $\phi(P)>\varepsilon_{A}$ for each $P \in A$. Then $\phi$ is strongly positive. Evidently every positive mapping is strongly positive. In a metric space, $d(P, Q)$ denotes the distance between $P$ and $Q$.

THEOREM 3.2. Let $K$ and $K^{\prime}$ be PL 3-manifolds, let $U$ be an open set in $|K|$, let $g$ be a homeomorphism $U \rightarrow\left|K^{\prime}\right|$, and let $\phi$ be a strongly positive function $U \rightarrow \mathbf{R}$. Then there is a homeomorphism $g^{\prime}: U \rightarrow\left|K^{\prime}\right|$ such that

(1) $g^{\prime}$ is $P L$ on every finite polyhedron in $U$ (relative to subdivisions of $K$ and $\left.K^{\prime}\right)$

(2) For each point $P$ of $U, d\left(g(P), g^{\prime}(P)\right)<\phi(P)$, and

(3) $g^{\prime}(U)=g(U)$.

This is Theorem 36.1, p. 253 of [MGT]. In the early literature, starting with $\left[\mathbf{M}_{5}\right]$, this theorem was freely used but never proved; it was not shown that $g^{\prime}$ could be chosen so that $g^{\prime}(U)=g(U)$.

Let $K$ be a complex, and let $S$ be a subset of $|K|$. Suppose that $S$ has a triangulation $K(S)$ such that every simplex of $K(S)$ is a linear subsimplex of some simplex of $K$. Then $S$ is a polyhedron, relative to $K$. If $S$ is compact, then each such $K(S)$ is finite, and $S$ is a finite polyhedron, relative to $K$. If $S$ is a polyhedron relative to $K$, and is closed, then $S$ forms a subcomplex of a subdivision of $K$, but for polyhedra in general this conclusion does not follow.

TheOREM 3.3. Let $K$ be a PL 3-manifold, let $U$ be an open subset of $|K|$, let $S$ be a subset of $U$, and suppose that $U$ has a triangulation $K(U)$ in which $S$ forms a subcomplex $K(S)$. Let $\phi$ be a strongly positive function $U \rightarrow \mathbf{R}$, and let $V$ be an open set such that $S \subset V \subset U$. Then there is a homeomorphism $g$ : $|K| \leftrightarrow|K|$ such that (1) $g(S)$ is a polyhedron relative to $K$, (2) for each point $P$ of $U, d(P, g(P))<\phi(P)$, and $(3) g \mid(|K|-V)$ is the identity.

This is a variation on Theorem 5 of $\left[\mathbf{M}_{5}\right]$. (Similarly for Theorem 3.4 below.) It is an easy consequence of Theorem 3.2. Define $\phi^{\prime}$ as a strongly positive function $V \rightarrow \mathbf{R}$ such that (a) $\phi^{\prime}(P) \leqslant \phi(P)$ for every $P$ and (b) for each $P, \phi^{\prime}(P)$ is less than the distance between $P$ and $|K|-V$. We can now apply Theorem 3.2 to the inclusion $i: V \leftrightarrow V, P \mapsto P$, using $K(U)$ as $K, K$ as $K^{\prime}$, and $\phi^{\prime}$ as $\phi$. By (b), the resulting PL approximation $i^{\prime}$ can be extended to $|K|-V$ as the identity.

In a $\mathrm{PL} 3$-manifold $K$, a set $S$ is tame (relative to $K$ ) if there is a 
homeomorphism $|K| \leftrightarrow|K|$, mapping $S$ onto a polyhedron (not necessarily a finite polyhedron). If there is an open set $U$ containing $S$, and a homeomorphism $U \rightarrow|K|$, mapping $S$ onto a polyhedron, then $S$ is semilocally tame. In the latter case it follows that $U$ has a triangulation $K(U)$ relative to which $S$ is a polyhedron. Thus Theorem 3.3 implies the following.

THEOREM 3.4. In a PL 3-manifold, every semi-locally tame set is tame. In fact, if $S$ is semilocally tame in $K$, and $V$ is an open set containing $S$, then there is a homeomorphism $g:|K| \leftrightarrow|K|$ such that (1) $g(S)$ is a polyhedron relative to $K$ and (2) $g \mid(|K|-V)$ is the identity.

Here it is not required that $S$ be compact, or even closed.

Theorem 3.5 (The Loop Theorem, first form; Ch. PapakyriakopouLOS). Let $K$ be an orientable PL 3-manifold with boundary, and let $M=|K|$. If there is a PL loop in $\mathrm{Bd} M$ which is contractible in $M$ but not in $\mathrm{Bd} M$, then there is a PL 2-cell $\Delta$ such that (1) $\mathrm{Bd} \Delta=\Delta \cap \mathrm{Bd} M$ and (2) $\mathrm{Bd} \Delta$ is not contractible in $\mathrm{Bd} M$.

Here a loop is a closed path without a distinguished base-point, that is, a mapping of a 1-sphere into a space. For proofs, see Papakyriakopoulos [P] and Stallings [St]. See also [MGT, pp. 182-190], where Stallings' proof is presented. This proof does not use orientability. The resulting generalized form of the Loop Theorem has the following consequence.

TheOREM 3.6 (The LoOP THEOREM, SECOND FORM). Let $K$ be a PL 3-manifold, let $M=|K|$, and let $M^{2}$ be a connected polyhedral 2-manifold which is a closed set in $M^{3}$, such that $M^{2}$ is 2-sided in $M^{3}$. Suppose that there is a PL loop in $M^{2}$ which is contractible in $M^{3}$ but not in $M^{2}$. Then there is a PL 2-cell $\Delta$ in $M^{3}$ such that (1) $\mathrm{Bd} \Delta=\Delta \cap M^{2}$ and (2) $\mathrm{Bd} \Delta$ is not contractible in $M^{2}$.

Here $M^{2}$ is 2-sided in $M^{3}$ if $M^{2}$ separates a connected open neighborhood of $M^{2}$. It is easy to show, geometrically, that under these conditions, if $U$ is such a connected neighborhood of $M^{2}$, then $U-M^{2}$ has exactly two components. Theorem 3.5 is simply a combination of Theorems 26.4 and 26.5 of [MGT]. If $M^{2}$ is the boundary of a polyhedral 3-manifold with boundary in $M^{3}$, then $M^{2}$ is always 2-sided in $M^{3}$, regardless of any question of orientability [MGT, Theorem 26.1, p. 191].

Note that in Theorem $3.5, M^{2}$ is not required to be compact.

4. Solid tori and toroidal shells. Of the results in this section, some are old, some must be folklore, and some may be new.

THEOREM 4.1. Let $M^{2}$ be a polyhedral 2-manifold, in a PL 3-manifold $K$. Let $D$ be a polyhedral 2-cell in $M^{2}$, and let $h$ be a PL homeomorphism $M^{2} \leftrightarrow M^{2}$, 
such that $h \mid\left(M^{2}-D\right)$ is the identity. Let $C$ be a polyhedral closed neighborhood of Int $D$. Then $h$ can be extended so as to give a PL homeomorphism $h^{\prime}$ : $|K| \leftrightarrow|K|$ such that $h^{\prime} \mid(|K|-C)$ is the identity.

See [MGT, Theorem 27.1, p. 197].

Hereafter in this section, $S$ will be a polyhedral solid torus, with $\mathrm{Bd} S=T$. Let $J$ be a 1 -sphere in Int $S$. If there is a homeomorphism

$$
\phi: \Delta \times S^{1} \leftrightarrow S
$$

(where $\Delta$ is a 2-cell and $S^{1}$ is a 1-sphere), such that $\phi\left(P \times S^{1}\right)=J$ for some $P \in$ Int $\Delta$, then $J$ is a spine of $S$. The following is obvious.

THEOREM 4.2. If $J$ is a spine of $S$, then $J$ carries a generator of the fundamental group $\pi(S)$; and if $J$ is a polygon, then $J$ carries a generator of the 1-dimensional homology group $H_{1}(S)$ (with coefficients in the group $\mathrm{Z}$ of integers).

By a polygon we mean a polyhedral 1 -sphere. If $J$ is a polygon in $T$, and $J$ carries a generator of $H_{1}(S)$, then $J$ is longitudinal in $S$. If $J$ bounds a 2-cell in $S$, but does not bound a 2-cell in $T$, then $J$ is latitudinal in $S$.

THEOREM 4.3. Let $J_{1}, J_{2}, \ldots, J_{n}$ be a collection of disjoint polygons in $T$. If $\cup_{i} J_{i}$ carries a generator of $H_{1}(S)$, then (1) some $J_{i}$ is longitudinal in $S$ and (2) every $J_{i}$ either is longitudinal in $S$ or bounds a 2-cell in $T$.

See [ $\mathbf{M}_{4}$, Lemmas 2 and 3]. Or see [MGT, Theorem 28.8, p. 204].

TheOREM 4.4. Let $A$ be a polyhedral annulus, and let $J$ be a polygon in Int $A$. Then (1) bounds a 2-cell in Int $A$ or (2) $J$ carries a generator of $H_{1}(A)$, and decomposes $A$ into two annuli $A_{1}, A_{2}$, containing the respective components of $\mathrm{Bd} A$, and intersecting in $J$.

This is a corollary of Theorem 27.1, p. 197 of [MGT].

THEOREM 4.5. Let $J_{1}$ and $J_{2}$ be disjoint polygons in $T$. If $J_{1}$ is latitudinal in $S$, then (1) $J_{2}$ is latitudinal in $S$ or (2) $J_{2}$ bounds a 2-cell in $T$.

INDICATION OF PROOF. Let $D$ be a polyhedral 2-cell in $S$, such that $J_{1}=\mathrm{Bd} D=D \cap T$. Split $S$ apart at $D$. This gives a 3-cell $C^{3}$, with $\mathrm{Bd} C^{3}$ the union of an annulus $A$ and two homeomorphic copies of $D$. If $J_{2}$ bounds a 2-cell in $A$, then (2) holds; if not, then (1) holds.

Let $J_{1}$ and $J_{2}$ be polygons in a polyhedral orientable 2-manifold $M^{2}$. If $J_{1} \cap J_{2}$ consists of a finite number of "true crossing points" of $J_{1}$ and $J_{2}$, then $J_{1}$ and $J_{2}$ are in general position (in $M^{2}$ ). We assign an orientation to $M^{2}$ (at random). To each polygon $J$ in $M^{2}$ we can assign an orientation (at random). This gives a 1-cycle $Z^{1}(J)$ with constant coefficient 1 . If $J_{1}$ and $J_{2}$ are in general position, then we can distinguish positive and negative crossings of $J_{1}$ 
and $J_{2}$. This gives the total crossing number $c=\operatorname{Cr}\left(Z^{1}\left(J_{1}\right), Z^{1}\left(J_{2}\right)\right)$. If we reverse the orientation of $M^{2}$, or $J_{1}$, or $J_{2}$, then $c$ is replaced by $-c$. Therefore $|c|$ is independent of the choices of these three orientations. If $|c|=1$, then we say that $J_{2}$ crosses $J_{1}$ algebraically once.

THEOREM 4.6. Let $J_{1}$ and $J_{2}$ be polygons in $T$, in general position. If $J_{1}$ is longitudinal and $J_{2}$ is latitudinal, then $J_{1}$ crosses $J_{2}$ algebraically once (and vice versa).

INDICATION OF PROOF. Let $D$ be a polyhedral 2-cell in $S$, such that $J_{2}=\operatorname{Bd} D=D \cap T$. As in the proof of Theorem 4.5, we split $S$ apart at $D$, getting a polyhedral 3-cell $C^{3}$, in which $T$ appears as an annulus $A \subset \operatorname{Bd} C^{3}$. The components of $J_{1} \cap \mathrm{Bd} C^{3}$ appear as disjoint broken lines $B \subset A$. If one of these has both its end-points in the same component of $\mathrm{Bd} A$, then it can be moved across $\mathrm{Bd} A$ into Int $A$ by a cellular PL homeomorphism $T \leftrightarrow T$; and this homeomorphism can be extended to $S$. Thus this operation reduces the number of points in $J_{1} \cap J_{2}$, preserving the stated properties of $J_{1}$ and $J_{2}$. Therefore we may suppose that each such $B$ has its end-points in different components of $\mathrm{Bd} A$. If there are $m$ such broken lines, then every 1-cycle $Z^{1}\left(J_{1}\right)$ (with constant coefficient 1 ) is homologous on $S$ to a cycle of the form $\pm m Z_{0}^{1}$, where $Z_{0}^{1}$ is a generator of $H_{1}(S)$. Since $J_{1}$ is longitudinal, it follows that $m=1$. The theorem follows.

THEOREM 4.7. Let $J$ be a latitudinal polygon in $S$, and let $J_{1}, J_{2}, \ldots, J_{m}$ be disjoint longitudinal polygons in $S$, such that $J_{i}$ and $J$ are in general position in $T$ for each $i$. Then there is a PL homeomorphism $g: S \leftrightarrow S$ such that each polygon $g\left(J_{i}\right)$ intersects $J$ in exactly one point (which is a "true crossing point").

INDICATION OF PROOF. When we split $T$ apart at $J, T$ becomes an annulus $A$, and each $J_{i}$ appears as finite union of disjoint broken lines $B$. As in the proof of the preceding theorem, the number of such broken lines can be reduced to 1 , for each $i$; and this can be done without increasing the total number of points in the intersections $J \cap J_{i}$. This can be done by a PL homeomorphism $S \leftrightarrow S$. The theorem follows.

TheOrem 4.8. Let $T$ be a PL torus, and let $J$ be a polygon in $T$ which does not bound a 2-cell in $T$. Then $T-J$ is homeomorphic to the interior of an annulus. Thus, if $T$ is split apart at $J$, giving a 2-manifold with boundary, the result is an annulus.

INDICATION OF PROOF. Theorem 28.7, p. 204 of [MGT] asserts that if $B$ is a regular neighborhood of such a $J$ in $T$, then $\mathrm{Cl}(T-B)$ is an annulus. Now we apply Theorem 4.4 to $J$ and the annulus $B$. 
Theorem 4.9. Let $S$ be a PL solid torus in a PL 3-manifold $M \subset \mathrm{S}^{3}$, and let $J$ be a polygon in $T=\mathrm{Bd} S$, such that $J$ is contractible in $M-\operatorname{Int} S$ but does not bound a 2-cell in $T$. Then $J$ is longitudinal in $S$.

Proof. By Theorem 3.2 we may suppose that the imbedding $M \rightarrow \mathrm{S}^{3}$ is PL. Let $A$ be an annulus which forms a regular neighborhood of $J$ in $T$, and let

$$
N=\left(\mathbf{S}^{3}-S\right) \cup \operatorname{Int} A,
$$

so that $N$ is a 3-manifold with boundary, and $\operatorname{Bd} N=\operatorname{Int} A$. Now $J$ is contractible in $(M-S) \cup \operatorname{Int} A$, and $(M-S) \cup$ Int $A \subset N$. By the Loop Theorem (first form) it follows that there is a PL 2-cell $\Delta$ in $N$, with $\Delta \cap \operatorname{Bd} N=\operatorname{Bd} \Delta$, such that $J^{\prime}=\mathrm{Bd} \Delta$ is not contractible in $N$. Using Theorems 4.1 and 4.4 we can show that there is a PL homeomorphism $N \leftrightarrow N, \operatorname{Bd} N \leftrightarrow \operatorname{Bd} N, J^{\prime} \leftrightarrow J$. Thus we may suppose that $\operatorname{Bd} \Delta=J$. Theorem 28.12, on p. 205 of [MGT], asserts that under these conditions, $J$ is longitudinal. (Note that without the hypothesis $M \subset \mathbf{S}^{3}$, this theorem would be trivially false; for example, it fails in every 3-manifold that contains a polyhedral projective plane.)

Let $T$ and $T^{\prime}$ be disjoint homeomorphic 2-manifolds, in a PL 3-manifold $K$. Suppose that there is a 3-manifold $W$ with boundary, lying in $|K|$, such that $\mathrm{Bd} W=T \cup T^{\prime}$, and $W$ is homeomorphic to $T \times[0,1]$. Then $T$ and $T^{\prime}$ are parallel (or concentric) (in $|K|$ ).

THEOREM 4.10. In an orientable PL 3-manifold $K$, let $F$ be a polygon. For $i=1,2$, let $T_{i}$ be a torus (not necessarily polyhedral) such that $T_{i}$ is the frontier of a closed neighborhood $S_{i}$ of $F$. Suppose that

(1) $S_{1}$ lies in the interior of a regular neighborhood $N$ of $F$ (relative to a subdivision of $K$ ),

(2) $S_{2} \subset S_{1}-T_{1}$, and,

(3) For $i=1,2$ there is an open set $V_{i}$ containing $T_{i}$, and a triangulation $K\left(V_{i}\right)$ of $V_{i}$, such that $T_{i}$ forms a subcomplex of $K\left(V_{i}\right)$.

Then

(4) For $i=1,2, S_{i}$ is a solid torus, with $F$ as a spine and

(5) $T_{1}$ and $T_{2}$ are parallel.

A set homeomorphic to $T \times[0,1]$ (where $T$ is a torus) will be called a toroidal shell.

Proof. By two applications of Theorem 3.3, the theorem reduces to the case in which $T_{1}$ and $T_{2}$ are polyhedra (relative to $K$ ). Let $S_{3}$ and $S_{4}$ be regular neighborhoods of $F$, in subdivisions of $K$, such that $S_{3} \subset$ Int $S_{2}$ and $S_{4} \subset$ Int $S_{3}$, and let $T_{i}=\operatorname{Bd} S_{i}(i=3,4)$. It is elementary to observe that Bd $N$ and $T_{3}$ are parallel, and that $T_{1}$ separates Bd $N$ from $T_{3}$ in $N-\operatorname{Int} S_{3}$. A special case of a theorem of C. H. Edwards ([E, p. 414, Theorem 9]) asserts 
that under these conditions $T_{1}$ is parallel to $\mathrm{Bd} N$ and to $T_{3}$. By repeated applications of Edwards' theorem it follows that there is a homeomorphism $N-$ Int $S_{4} \leftrightarrow \operatorname{Bd} N \times[0,1]$, mapping each set $\mathrm{Bd} N, T_{i}$ onto a slice $\mathrm{Bd} N \times$ $k$. Therefore, for $i=1,2$ there is a homeomorphism $g_{i}: N \leftrightarrow S_{i}$ such that $g_{i} \mid S_{4}$ is the identity. Thus (4) holds. Similarly, there is a homeomorphism $h$ : $S_{1} \leftrightarrow N, S_{2} \leftrightarrow S_{3}$, so that (5) holds.

I am indebted to the referee for the reference to Edwards, and for the scheme of the above proof.

Theorem 4.11. In a PL 3-manifold $M$, let $A$ be a polyhedral annulus, let $J$ and $J^{\prime}$ be polygons in Int $A$, each of which separates the components of $\mathrm{Bd} A$ from one another in $A$, and let $W$ be a neighborhood of Int $A$ in $M$. Then there is a PL homeomorphism $h: M \leftrightarrow M, A \leftrightarrow A, J \leftrightarrow J^{\prime}$, such that $h \mid(M-W)$ and $h \mid \mathrm{Bd} A$ are identity mappings.

Proof. Theorem 27.2, p. 197 of [MGT], asserts that under these conditions we can move $J$ onto $J^{\prime}$ by a PL homeomorphism $h^{\prime}$ such that $h^{\prime} \mid \operatorname{Bd} A$ is the identity and $h^{\prime}$ is the composition of a finite sequence of cellular homeomorphisms. Now we use Theorem 4.1 repeatedly to get the desired $h$.

The following is needlessly special, but is sufficient for our present purposes.

THEOREM 4.12. Let $J$ be a polygon, in a polyhedral torus $T$, such that $J$ does not bound a 2-cell in $T$. Then $J$ is not contractible in $T$.

Proof. Let $S$ be a polyhedral solid torus, with $\operatorname{Bd} S=T^{\prime}$. By Theorem 4.8 we know that when $T$ is split apart at $J$, the result is an annulus $A$. Therefore there is a mapping $h: A \rightarrow T^{\prime}$, such that $h$ "reidentifies the components of Bd $A$," and maps these onto a longitudinal polygon $J$ in $T^{\prime}$. Thus there is a homeomorphism $T \leftrightarrow T^{\prime}, J \leftrightarrow J^{\prime}$. Since $J^{\prime}$ is not contractible in $T^{\prime}, J$ is not contractible in $T$.

Note that the proof proves more than the theorem; it shows that a polygon can be imbedded in a PL torus in only two topologically different ways.

5. Invariant toroidal neighborhoods of $F$. Let $M \subset \mathbf{S}^{3}, f, F$, and $n$ be as in Theorem 1.1. By Theorem 1.3 we know that $f$ has period exactly $n$ at each point of $M-F$. Let $\Omega$ be the orbit space of $f$, with the usual topology, and let

$$
\operatorname{Pr}: M \rightarrow \Omega
$$

be the projection. Let $K(M)$ be a triangulation of $M$ in which $F$ forms a subcomplex. Let

$$
\tilde{U}=M-F, \quad U=\operatorname{Pr} \tilde{U}, \quad \rho=\operatorname{Pr} \mid \tilde{U} .
$$

Then $\rho: \tilde{U} \rightarrow U$ is an $n$-sheeted covering. Since $U$ is a 3 -manifold, it follows that $U$ has a triangulation $K(U)$. We may suppose that the diameters 
$\delta \sigma$ of the simplices $\sigma$ of $K(U)$ approach 0 as $\sigma$ approaches $\operatorname{Pr} F$, in the sense that for every $\varepsilon>0$ there is a neighborhood $N_{\varepsilon}$ of $\operatorname{Pr} F$ such that if $\sigma$ intersects $N_{\varepsilon}$, then $\delta \sigma<\varepsilon$. (This property is obtainable by subdivision. Deductively, it will not be needed, but to omit it might seem unnatural.)

Let $K(\tilde{U})$ be the lifting of $K(U)$, that is, the set of all components of all sets of the form $\rho^{-1}(\sigma)$, where $\sigma \in K(U)$. Then $K(\tilde{U})$ is a triangulation of $\tilde{U}$, and $f \mid \tilde{U}$ is simplicial relative to $K(\tilde{U})$. Note that we have no reason to suppose that the simplices of $K(\tilde{U})$ are polyhedral relative to $K(M)$.

A subset $A$ of $M$ will be called $f$-invariant if $f(A)=A$.

THEOREM 5.1. Every open neighborhood $X$ of $F$ contains a solid torus $\tilde{S}_{X}$ such that (1) $\tilde{S}_{X}$ is f-invariant, (2) Bd $\tilde{S}_{X}$ is polyhedral relative to $K(\tilde{U})$, and (3) $F$ is a spine of $\tilde{S}_{X}$.

For an indication of the way in which this theorem will be used, see the remarks just after the proof.

Proof. Evidently there is no loss of generality in supposing that $\bar{X}$ is a regular neighborhood of $F$ in a subdivision of $K(M)$. Let $S$ be a regular neighborhood of $F$, in a subdivision of $K(M)$, chosen sufficiently small so that

$$
\bigcup_{i} f^{i}(S) \subset X
$$

We assert that $\operatorname{Pr} F$ has arbitrarily small closed connected neighborhoods $S_{X}$ such that $T=\operatorname{Fr} S_{X}$ is a 2-manifold which is polyhedral relative to $K(U)$. (For example, take a small closed neighborhood $W$ of $\operatorname{Pr} F$, such that $\operatorname{Fr} W$ is a polyhedron, and then add to $W$ a small regular neighborhood of Fr $W$. Note that we write Fr $S_{X}$, not Bd $S_{X}$ : not for a long time will we know that $S_{X}$ is a 3-manifold with boundary.) If $T$ is not connected, then we can make it connected, simply by boring holes in $S_{X}-\operatorname{Pr} F$, from one component of $T$ to another. Thus we may suppose that

(a) $T=\operatorname{Fr} S_{X}$ is a connected 2-manifold which is polyhedral relative to $K(U)$.

We choose $S_{X}$ so that (b) $T \subset$ Pr Int $S$ and (c) the set

$$
T-\operatorname{Pr} \bigcap_{i} f^{i}(\operatorname{Int} S)
$$

lies in a finite union of disjoint 2-cells $D_{j}$. (At the outset, we get (b) and (c) simply by requiring that $\left(\mathrm{b}^{\prime}\right) T \subset \operatorname{Pr} \cap{ }_{i} f^{i}\left(\right.$ Int $S$ ). But $\left(\mathrm{b}^{\prime}\right)$ would not necessarily be preserved by certain hypothetical operations presently to be described.) Evidently $T$ is 2-sided in $U$, because $S_{X}-\operatorname{Pr} F$ is a 3-manifold with boundary, and $T$ is its frontier in $U$ [MGT, Theorem 26.1, p. 191].

Suppose that $\Delta$ is a polyhedral 2-cell in $\operatorname{Pr}(\operatorname{Int} S-F)$, such that $\operatorname{Bd} \Delta=\Delta$ $\cap T$ and $\mathrm{Bd} \Delta$ is not contractible in $T$. Then $\Delta$ will be called a Loop Theorem 
Disk (LTD) in the pair [Pr $S, T]$. If there is such an LTD $\Delta$, then $\Delta$ can be chosen so as not to intersect any of the 2-cells $D_{j}$; any given $\Delta$ can be moved off the $D_{j}$ 's, preserving its stated properties. If $\Delta \cap D_{j}=\varnothing$ for each $j$, then $\Delta$ can be added to $T$, and the resulting 2-dimensional polyhedron can be split apart at $\Delta$, giving a 2-manifold $T^{\prime}=$ Fr $S_{X}^{\prime}$, where $S_{X}^{\prime}$ is a neighborhood of $\operatorname{Pr} F$. There are now two cases to consider.

Case 1. $T^{\prime}$ is connected. Here $S_{X}^{\prime}$ is smaller or larger than $S_{X}$, according as $\Delta \subset S_{X}$ or Int $\Delta \cap S_{X}=\varnothing$. In either case, the 1-dimensional Betti number $p^{1}\left(T^{\prime}\right)$ (with integer coefficients) is $p^{1}(T)-2$.

Case 2. $T^{\prime}$ is not connected, $=T_{1} \cup T_{2}$. Since $\operatorname{Pr}^{-1}\left(T^{\prime}\right)$ separates $F$ from Bd $S$ in $S$, some component $C$ of $\operatorname{Pr}^{-1}\left(T^{\prime}\right)$ has the same property. Let $U$ be the component of $S-C$ that contains $F$, and let $S_{X}^{\prime \prime}=\operatorname{Pr} \bar{U}$. Then $\operatorname{Fr} S_{X}^{\prime \prime}$ is $T_{1}$ or $T_{2}$, say, $T_{1}$; and since $T_{2}$ is not a 2-sphere, we have $p^{1}\left(T_{1}\right)<p^{1}(T)-2$. We replace $S_{X}$ by $S_{X}^{\prime \prime}$, and $T$ by $T_{1}$.

Under the above conditions for $\Delta$, the splitting operation preserves not only (a) and (b) but also (c). And iterations of the operation must terminate. Thus we may assume hereafter that we have $S_{X}$ and $T$ satisfying (a), (b), (c), and also

(d) The pair $[\operatorname{Pr} S, T]$ contains no LTD.

Let

$$
\tilde{S}_{X}=\operatorname{Pr}^{-1} S_{X}, \quad \tilde{T}=\operatorname{Pr}^{-1} T=\operatorname{Bd} \tilde{S}_{X}
$$

LEMMA 5.1.1. $\tilde{S}_{X} \subset X$.

Proof. Since $T \subset \operatorname{Pr}$ Int $S$, we have

$$
\tilde{T}=\operatorname{Pr}^{-1} T \subset \operatorname{Pr}^{-1} \operatorname{Pr} \text { Int } S=\bigcup_{i} f^{i}(\text { Int } S) \subset X .
$$

Therefore $\tilde{S}_{X} \subset X$.

LEMMA 5.1.2. $\tilde{T}$ is connected.

Proof. $\tilde{S}_{X}$ is connected, because every component of $\tilde{S}_{X}$ contains $F$. Since $\bar{X}$ is a regular neighborhood of a polygon, $M-X$ is connected. Let $Y$ be the component of $M-\tilde{S}_{X}$ that contains $M-X$. Since $\tilde{S}_{X}$ is $f$-invariant, so also is $\bar{Y} ; \bar{Y}$ is a PL 3-manifold with boundary; and $\mathrm{Bd} \bar{Y} \subset \tilde{T}$. Since $\tilde{S}_{X}$ is connected, so also is $\mathrm{Bd} \bar{Y}$; $\mathrm{Bd} \bar{Y}$ is a component of $\tilde{T}$, and is $f$-invariant. Thus $\operatorname{Pr} \operatorname{Bd} \bar{Y}$ is a component of $T$. If $B$ were another component of $\tilde{T}$, then $\operatorname{Pr} B$ would be another component of $T$, which is impossible, because $T$ is connected. Therefore $\tilde{T}$ is connected, which was to be proved.

LEMMA 5.1.3. Let $i$ be the inclusion $\tilde{T} \rightarrow X-F$. Then the induced homomorphism $i^{*}: \pi(\tilde{T}) \rightarrow \pi(X-F)$ is injective. 
Proof. Let $\tilde{p}$ be a closed path in $\tilde{T}$, and let $p=\operatorname{Pr}(\tilde{p})$. Let $\left\{D_{j}\right\}$ be as in condition (c) above. Then $p$ can be moved, by a homotopy, to give a closed path $p^{\prime}$ in $T \cap \operatorname{Pr} \cap{ }_{i} f^{i}$ (Int $\left.S\right)$, and the homotopy between $p$ and $p^{\prime}$ in $\pi(T)$ can be lifted so as to give a closed path $\tilde{p}^{\prime}$, in $\tilde{T} \cap \cap_{i} f^{i}(\operatorname{Int} S)$, such that $\tilde{p}^{\prime} \simeq \tilde{p}$ in $\pi(\tilde{T})$.

Thus, if the lemma is false, there is a closed path $\tilde{p}^{\prime}$ in $\tilde{T} \cap \cap_{i} f^{i}($ Int $S$ ) such that $\tilde{p}^{\prime}$ is contractible in $X$ but not in $\tilde{T}$. Since $\bar{X}$ and $S$ are regular neighborhdoods of $F$ in $K$, with $S \subset X$, it follows that $S$ is a retract of $\bar{X}$. (Use Theorem 4.10 , or some much simpler theorem.) Therefore $\tilde{p}^{\prime}$ is contractible in $S-F$, and hence in Int $S-F$. Therefore $p^{\prime}=\operatorname{Pr}\left(\tilde{p}^{\prime}\right)$ is contractible in $\operatorname{Pr}(\operatorname{Int} S-F)$. But since $(\operatorname{Pr} \mid T)^{*}: \pi(\tilde{T}) \rightarrow \pi(T)$ is injective, $p$ is not contractible in $T$. But since $T=\operatorname{Fr} S_{X}$, it follows that $T$ is 2-sided in the 3-manifold $\operatorname{Pr}(\operatorname{Int} S-F)$. Therefore Theorem 3.5 applies, and gives us a contradiction of condition (d) for $S$ and $T$.

LEMMA 5.1.4. $\tilde{T}$ is a torus.

Proof. Since $\pi(X-F) \approx \pi(\operatorname{Fr} X) \approx \mathbf{Z}+\mathbf{Z}$, it follows by the preceding lemma that $\pi(\tilde{T})$ is commutative. Therefore $T$ is either a torus or a 2 -sphere. Now $\tilde{T}$ is polyhedral relative to $K(\tilde{U})$. By Theorem 3.3 (as in the proof of Theorem 4.10), there is a homeomorphism $h: M \leftrightarrow M$ such that $h(\tilde{T})$ is a polyhedron relative to $K(M)$ and $h$ is the identity except at points of $X-F$. Thus, if $\tilde{T}$ is a 2 -sphere, then $F$ lies in a 3-cell in $X$, which is impossible. The lemma follows.

By Theorem 4.10 it follows that $\tilde{S}_{X}$ is a solid torus. This completes the proof of Theorem 5.1.

Nearly all of the rest of this paper will be devoted to the proof of Theorem 11.5, which asserts, in effect, that the $\tilde{S}_{X}$ of Theorem 5.1 can be chosen so that $\operatorname{Pr} \tilde{S}_{X}$ is a solid torus. (The $S_{1}$ of Theorem 11.5 is a suitably chosen $\tilde{S}_{X}$ as in Theorem 5.1.) In the proof of Theorem 11.5 we form a "standard" solid torus $S_{1}^{\prime}$, and decompose it into an infinite collection of solid tori, plus a spine $F^{\prime}$. Thus the purpose of $\$ \S 6-11$ below is to define a neighborhood $S_{1}$ of $F$ such that $\operatorname{Pr} S_{1}$ has a similar decomposition; this will enable us to define the desired homeomorphism in the proof of Theorem 11.5. The proof of Theorem 1.1 will then become easy.

6. Eliminating cellular oscillations. We resume the proof of Theorem 1.1. In $\S \S 6-11$, the definitions and notations of $\S 5$, up to and including Theorem 5.1, will be regarded as conventions, and used without reference or comment.

The next step in the proof of Theorem 1.1 is to show that every neighborhood of $F$ contains a solid torus $S$, satisfying the conditions for $\tilde{S}_{X}$ in Theorem 5.1, such that $S$ "approximates the shape of a regular neighborhood of $F$." A usable definition of the latter idea will emerge in this and later 
sections. Meanwhile it is intuitively evident that a solid-toroidal neighborhood of $F$ may fail, in at least two ways, to have the desired property.

(a) If $J$ is a polygon in the boundary of a regular neighborhood $N$ of $F$, and $J$ is of small diameter, and bounds a 2-cell in Bd $N$, then $J$ bounds a 2-cell of small diameter in $\mathrm{Bd} N$. If $S$ fails to have this property, then we say that $\mathrm{Bd} S$ has cellular oscillations.

(b) If $J$ and $J^{\prime}$ are disjoint latitudinal polygons in $\operatorname{Bd} N$, and $J \cup J^{\prime}$ is of small diameter, then $J \cup J^{\prime}$ bounds an annulus of small diameter in $\mathrm{Bd} N$. If $S$ fails to have this property, then we say that $\mathrm{Bd} S$ has annular oscillations.

We shall show, in effect, that these are the only possible difficulties, and that they can be dealt with separately, in the stated order.

Let $N$ be a regular neighborhood of $F$, relative to a subdivision $K^{\prime}(M)$ of $K(M)$. Then $N$ has a natural decomposition into dual cells $D_{v}$, which are neighborhoods of the points $v$ of $F$ which are vertices of $K^{\prime}(M)$, such that

(1) Two sets $D_{v}, D_{w}$ intersect only if $v$ and $w$ are the end-points of an edge of $K^{\prime}(M)$ and

(2) Each nonempty intersection $D_{v} \cap D_{w}=E_{v w}$ is a PL 2-cell, lying in $\mathrm{Bd} D_{v} \cap \mathrm{Bd} D_{w}$ and intersecting $F$ in exactly one point.

The 2-cells $E_{v w}$ are "orthogonal to the edges $v w . "$ They are called splitting disks.

Let $N_{0}$ and $N_{1}$ be regular neighborhoods of $F$, defined relative to subdivisions $K^{\prime}(M)$ and $K^{\prime \prime}(M)$ of $K(M)$. Let

$$
\mathbf{P}=\left\{P_{1}, P_{2}, \ldots, P_{m}\right\}
$$

be a finite set of vertices of $K^{\prime}(M)$, lying in $F$, such that the points $P_{j}$ appear in the stated cyclic order on $F$. (We use integers modulo $m$ as subscripts, here and in similar situations hereafter.) Suppose that no two points of $\mathbf{P}$ are consecutive on $F$ in $K^{\prime}(M)$, so that the dual cells $D_{j}=D_{P_{j}}$ of $N_{0}$ that contain the points $P_{j}$ are disjoint. For each $j$, let $D_{j}^{\prime}$ be the dual cell of $N_{1}$ that contains $P_{j}$, and suppose that $K^{\prime \prime}(M)$ is a subdivision of $K^{\prime}(M)$, sufficiently fine so that $N_{1} \subset \operatorname{Int} N_{0}$, and

$$
\bigcup_{i} f^{i}\left(D_{j}^{\prime}\right) \subset \bigcap_{i} f^{i}\left(\operatorname{Int} D_{j}\right)
$$

for each $j$. It is geometrically clear that for each $j$ there is a polyhedral 2-cell $E_{j} \subset D_{j}$ such that (1) $\mathrm{Bd} E_{j}=E_{j} \cap \mathrm{Bd} D_{j}$, (2) $E_{j}$ intersects no splitting disk of $N_{0}$ or $N_{1}$, (3) $E_{j} \cap \mathrm{Bd} D_{j}^{\prime}$ is a polygon, and (4) $E_{j} \cap F=P_{j}$. Thus $E_{j}$ separates $D_{j}$ (and $D_{j}^{\prime}$ ) into two disjoint connected sets each of which contains a point of $F \cap \operatorname{Bd} D_{j}$ (and of $\left.F \cap \operatorname{Bd} D_{j}^{\prime}\right)$. For each $j$, let

$$
B_{j}=E_{j}-\text { Int } D_{j}^{\prime} \text {. }
$$

Then $B_{j}$ is an annulus; the components of $\mathrm{Bd} B_{j}$ are polygons lying in $\operatorname{Bd} N_{0} \cap \operatorname{Bd} D_{j}$ and $\mathrm{Bd} N_{1} \cap \mathrm{Bd} D_{j}^{\prime}$, and intersecting no splitting disk of $N_{0}$ 
or $N_{1}$, and such that

$$
\text { Int } B_{j} \subset \text { Int } D_{j}-D_{j}^{\prime} \text {. }
$$

So far, all the sets mentioned are polyhedra relative to $K(M)$, but are not necessarily polyhedra relative to $K(\tilde{U})$; many of them are not even subsets of $\tilde{U}$.

Let $K_{1}(\tilde{U})$ be a triangulation of $\tilde{U}$, such that every simplex of $K_{1}(\tilde{U})$ is a linear subsimplex of some simplex of $K^{\prime \prime}(M)$, and such that $\delta \sigma\left(\sigma \in K_{1}(U)\right)$ approaches 0 as $\sigma \rightarrow F$. Let $\phi: U \rightarrow \mathbf{R}$ be a strongly positive function. By Theorem 3.3 it follows that there is a homeomorphism

$$
g: \tilde{U} \leftrightarrow \tilde{U}
$$

such that $g$ is PL relative to $K_{1}(\tilde{U})$ and $K(\tilde{U})$, and such that $g$ is a $\phi$-approximation of the identity, in the sense that for each point $P$ we have $d(P, g(P))<\phi(P)$. If $\phi$ is chosen so that $\phi(P)$ is always less than the distance from $P$ to $F(=M-\tilde{U})$, then $g$ can be extended to give a homeomorphism

$$
g: M \leftrightarrow M,
$$

such that $g \mid F$ is the identity. For each $j$, let

$$
C_{j}=g\left(D_{j}\right), \quad C_{j}^{\prime}=g\left(D_{j}^{\prime}\right), \quad A_{j}=g\left(B_{j}\right), \quad d_{j}=g\left(E_{j}\right), \quad S_{0}=g\left(N_{0}\right) .
$$

We take $\phi$ sufficiently small so that $g$ preserves the stated relation between the $D_{j}$ 's and the $D_{j}^{\prime \prime}$; that is,

$$
\bigcup_{i} f^{i}\left(C_{j}^{\prime}\right) \subset \bigcap_{i} f^{i}\left(C_{j}\right)
$$

Let

$$
\mathbf{C}=\left\{C_{j}\right\}, \quad \mathbf{C}^{\prime}=\left\{C_{j}^{\prime}\right\}, \quad \mathbf{A}=\left\{A_{j}\right\}
$$

Then the system

$$
\mathbf{B}=\left[\mathbf{P}, \mathbf{C}, \mathbf{C}^{\prime}, \mathbf{A}, S_{0}\right]
$$

is a barrier system for $F$. The mesh $\delta \mathbf{B}$ of $\mathbf{B}$ is the maximum of the diameters of the sets $C_{j}$ and the diameters of the components of $S_{0}-\cup_{j} C_{j}$. Evidently the preceding discussion has proved nearly all of the following:

THEOREM 6.1. Let $N$ be a regular neighborhood of $F$, and let $\varepsilon$ be a positive number. Then there is a barrier system

$$
\mathbf{B}=\left[\mathbf{P}, \mathbf{C}, \mathbf{C}^{\prime}, \mathbf{A}, S_{0}\right]
$$

for $F$ such that (1) $S_{0} \subset N$ and (2) $\delta \mathbf{B}<\varepsilon$. And given any finite subset $\mathbf{Q}$ of $F$, $\mathbf{B}$ can be chosen so that (3) $\mathbf{Q} \subset \mathbf{P}$.

(To get this, we choose $\mathbf{P}$ so that $\mathbf{Q} \subset \mathbf{P}$, and take the points of $\mathbf{P}$ sufficiently close together so that the components of $F-\mathbf{P}$ have diameter 
less than $\varepsilon$. Then form $N_{0} \subset N$, using a very fine subdivision $K^{\prime}(M)$ of $K(M)$, and take $\phi$ very close to 0 .)

Definition 6.2. Let $\mathbf{B}=\left[\mathbf{P}, \mathbf{C}, \mathbf{C}^{\prime}, \mathbf{A}, S_{0}\right]$ be a barrier system for $F$. Let $S$ be a solid torus such that

(1) $F \subset$ Int $S, S \subset$ Int $S_{0}$,

(2) $\mathrm{Bd} S$ is polyhedral relative to $K(\tilde{U})$,

(3) $S \cap \cup_{j} A_{j}=\varnothing$, and

(4) $\mathrm{Bd} S$ is in general position relative to each set Bd $C_{j}^{\prime}$, in the sense that $\mathrm{Bd} S \cap \mathrm{Bd} C_{j}^{\prime}$ is a finite union of disjoint polygons, at which $\mathrm{Bd} S$ and Bd $C_{j}^{\prime}$ pierce each other locally in $\tilde{U}$. Then $\mathbf{B}$ is a barrier system for $S$.

THEOREM 6.3. Let $\mathrm{B}$ be a barrier system for $S$. Then each set $\mathrm{Bd} S \cap \mathrm{Bd} C_{j}^{\prime}$ contains a polygon $J$ which is latitudinal in $S$.

Proof. Bd $C_{j}^{\prime} \cap \mathrm{Bd} A_{j}$ separates $\mathrm{Bd} C_{j}^{\prime}$ into two disjoint sets each of which is the interior of a 2-cell. Since $S \cap A_{j}=\varnothing$, it follows that the two points $P$, $Q$ of $F \cap \operatorname{Bd} C_{j}^{\prime}$ lie in different components of $S \cap \mathrm{Bd} C_{j}^{\prime}$.

Let $M^{2}$ be the union of (1) the component $W$ of $S \cap \operatorname{Bd} C_{j}^{\prime}$ that contains $P$ and (2) all components of $S \cap \mathrm{Bd} C_{j}^{\prime}$ that lie in components of $\mathrm{Bd} C_{j}^{\prime}-W$ that do not contain $Q$. Evidently $W$ is a 2-cell with a finite number of holes (perhaps none). Let $J$ be the "outer boundary" of $W$, that is, the component of $\mathrm{Bd} W$ that is the boundary of the closure of the component of $\mathrm{Bd} C_{j}^{\prime}-W$ that contains $Q$. We shall show that $J$ is latitudinal in $S$.

LEMMA 6.3.1. Every component of $\mathrm{Bd} M^{2}$, other than $J$, bounds a 2-cell in Bd $S$.

Proof. $J$ is the boundary of a 2-cell $D_{J} \subset \mathrm{Bd} C_{j}^{\prime}$, with $M^{2} \subset D_{J}$. Suppose that the lemma is false, so that some component $J^{\prime}$ of $\mathrm{Bd} M^{2}-J$ does not bound a 2-cell in $\mathrm{Bd} S$; and choose $J^{\prime}$ as the inmost polygon in $D_{J}$ with the stated properties. It follows that $J^{\prime}$ is the boundary of a 2-cell $d$ (PL relative to $K(\tilde{U})$ ) such that Int $d$ lies in either Int $S-F$ or Int $S_{0}-S$. The point is that $J^{\prime}$ is the outer boundary of a 2-cell with holes whose interior lies in Int $S-F$ or in Int $S_{0}-S$; and since the boundaries of the holes bound 2-cells in $\mathrm{Bd} S$, it follows that the holes can be filled, with 2-cells lying arbitrarily close to $\mathrm{Bd} S$, so as to give the desired $d$.

By Theorem 4.10 we know that $F$ is a spine of $S$ and of $S_{0}$. Suppose that Int $d \subset \operatorname{Int} S-F$. Since $J^{\prime}$ does not bound a 2-cell in $\mathrm{Bd} S$, it follows that $J^{\prime}$ is latitudinal in $S$, which is impossible, because $d \cap F=\varnothing$. Suppose that Int $d \subset$ Int $S_{0}-S$. Then it follows by Theorem 4.9 that $J^{\prime}$ is longitudinal in $S$. Therefore $J^{\prime}$ carries a generator of $\pi(S)$, and the injection $\pi(S) \rightarrow \pi\left(S_{0}\right)$ annihilates $\pi(S)$. This is absurd, because $F$ is a spine both of $S$ and of $S_{0}$.

From the lemma it follows that $J$ is the boundary of a 2-cell $d$, such that 
Int $d \subset S$; the proof is the same as that of the lemma. (Every component of Bd $M-J$ bounds a 2-cell in Bd $S$, and so the holes in $M$ can be filled with 2-cells lying in Int $S$.) Therefore $J$ is latitudinal in $S$, which was to be proved.

TheOREM 6.4. Let $\mathbf{B}=\left[\mathbf{P}, \mathbf{C}, \mathbf{C}^{\prime}, \mathbf{A}, S_{0}\right]$ be a barrier system for $S$. Then every polygon $J$ which lies in a set $\mathrm{Bd} S \cap C_{i}$ either is latitudinal in $S$ or bounds $a$ 2-cell in Bd $S$.

Proof. Take $j \neq i$, and let $J_{j}$ be a polygon in $\mathrm{Bd} S \cap \mathrm{Bd} C_{j}^{\prime}$ such that $J_{j}$ is latitudinal in $S$. Let $D_{j}$ be a 2-cell such that $\mathrm{Bd} D_{j}=J_{j}$ and Int $D_{j} \subset$ Int $S$. When we "split $S$ apart at $D_{j}$," we get a 3-cell $C$ whose boundary is the union of two 2-cells $D_{j}^{\prime}, D_{j}^{\prime \prime}$ and an annulus $A$ whose interior contains $J$. If $J$ bounds homologically on $A$, then $J$ bounds a 2-cell in $A$, and hence bounds a 2-cell in Bd $S$. If $J$ separates $D_{j}^{\prime}$ from $D_{j}^{\prime \prime}$ in $\mathrm{Bd} C$, then $J$ is latitudinal in $S$.

THEOREM 6.5. Let $\mathbf{B}=\left[\mathbf{P}, \mathbf{C}, \mathbf{C}^{\prime}, \mathbf{A}, S_{0}\right]$ be a barrier system for $F$. Then every neighborhood $N$ of $F$ contains a solid torus $S$ such that

(1) $S$ if $f$-invariant, and $\mathrm{Bd} S$ is polyhedral relative to $K(\tilde{U})$,

(2) B is a barrier system for $S$, and

(3) If $J$ is a polygon in a set $\mathrm{Bd} S \cap C_{j}^{\prime}$, and $J$ bounds a 2-cell in $\mathrm{Bd} S$, then $J$ bounds a 2-cell in $\mathrm{Bd} S \cap \cap, f^{r}\left(\right.$ Int $\left.C_{j}\right)$.

Proof. First take $S$ as a solid torus $\tilde{S}_{X}$ as in Theorem 5.3. Thus (1) is satisfied. If $S$ lies in a sufficiently small neighborhood of $F$, then $S \subset N$, and conditions (1), (2), and (3) of Definition 6.2 are satisfied. To get (4), we move $\operatorname{Pr} \operatorname{Bd} S$ slightly, so that no edge of $\operatorname{Pr} \operatorname{Bd} S$ intersects any set $\operatorname{Pr} e$, where $e$ is an edge of a set $\mathrm{Bd} C_{j}^{\prime}-F$; we then lift by $\operatorname{Pr}^{-1}$ to get a new $S$ which satisfies (1)-(4) of Definition 6.2.

It remains to show that $S$ can be chosen so that (3) of Theorem 6.5 is also satisfied. For each $j$, let $P_{j}^{-}$and $P_{j}^{+}$be the points of $F \cap \mathrm{Bd} C_{j}^{\prime}$, such that $P_{j-1}, P_{j-1}^{+}, P_{j}^{-}, P_{j}, P_{j}^{+}$appear in the stated cyclic order on $F$. The points $P_{j}^{-}$, $P_{j}^{+}$lie in the interiors of 2-cells ${D_{j}}^{-}, D_{j}^{+}$, lying in Bd $C_{j}^{\prime}$, such that

$$
\bigcup_{i} f^{i}\left(D_{j}^{-}\right), \bigcup_{i} f^{i}\left(D_{j}^{+}\right) \subset N
$$

and

$$
\bigcup_{i} f^{i}\left(D_{j}^{-}\right) \cap \bigcup_{k} A_{k}=\bigcup_{i} f^{i}\left(D_{j}^{+}\right) \cap \bigcup_{k} A_{k}=\varnothing .
$$

(Any sufficiently small 2-cell neighborhoods of ${P_{j}^{-}}^{-}$and $P_{j}^{+}$in $\mathrm{Bd} C_{j}^{\prime}$ will do.) Note that under the conditions for a barrier system for $F$, we automatically have

$$
f^{i}\left(D_{j}^{-}\right), f^{i}\left(D_{j}^{+}\right) \subset C_{j} \subset S_{0},
$$

and $f^{i}\left(D_{j}^{-}\right) \cap A_{k}=f^{i}\left(D_{j}^{+}\right) \cap A_{k}=\varnothing$ for $j \neq k$. Then we choose $S$, subject 
to all the above conditions, such that

$$
\text { Bd } S \cap \operatorname{Bd} C_{j}^{\prime} \subset \text { Int } D_{j}^{-} \cup \text { Int } D_{j}^{+}
$$

for each $j$.

For each $j$, consider the set

$$
Y=\operatorname{Pr}\left[\operatorname{Bd} S \cap \bigcup_{r} f^{r}\left(C_{j}^{\prime}\right)\right] .
$$

This is a finite polyhedron (relative to $K(U)$ ) in $\Omega-\operatorname{Pr} F$. Let $X_{j}$ be a compact polyhedral 2-manifold with boundary which forms a neighborhood of $Y$ in $\operatorname{Pr} \operatorname{Bd} S$, sufficiently small so that

$$
X_{j} \subset \operatorname{Pr} \bigcap_{r} f^{r}\left(\operatorname{Int} C_{j}\right)
$$

and let

$$
\tilde{X}_{j}=\operatorname{Pr}^{-1} X_{j}
$$

Then $\tilde{X}_{j}$ is also a compact polyhedral 2-manifold with boundary. Let $p_{j}^{1}$ be the 1-dimensional Betti number of $X_{j}$ (with integers modulo 2 as coefficients). Since each $p_{j}^{1}$ is finite, we may assume, subject to all the above conditions, that $S$ and $X_{j}$ are chosen so as to minimize $\Sigma p_{j}^{1}$. On this basis we shall show that $S$ satisfies condition (3) of Theorem 6.5.

Suppose that (3) does not hold, and let $J$ be a polygon, with $J \subset \operatorname{Bd} S \cap$ $C_{j}^{\prime}$, such that $J$ bounds a 2-cell $D$ in Bd $S$, but $J$ does not bound a 2-cell in

$$
\text { Bd } S \cap \bigcap_{r} f^{r}\left(\text { Int } C_{j}\right) \text {. }
$$

By general position, $D \cap \mathrm{Bd} C_{j}^{\prime}$ is a finite union of disjoint polygons $J_{i}$. Since each $J_{i}$ bounds a 2-cell in $\mathrm{Bd} S$, it follows that no $J_{i}$ separates $P_{j}^{-}$from $P_{j}^{+}$in Bd $C_{j}^{\prime}$. Therefore each $J_{i}$ bounds a 2-cell $D_{i}$ in $\mathrm{Bd} C_{j}^{\prime}-F$; and since $J_{i}$ lies in $D_{j}^{-}$or $D_{j}^{+}$, so also does $D_{i}$. Evidently $J_{i} \subset \tilde{X}_{j}=\operatorname{Pr}^{-1} X_{j}$. If each $J_{i}$ bounds a 2-cell in $\tilde{X}_{j}$, then it follows that

$$
D \subset \tilde{X}_{j} \subset \operatorname{Bd} S \cap \bigcap_{r} f^{r}\left(\text { Int } C_{j}\right),
$$

which contradicts our assumption for $J$. Therefore some $J_{i}$ does not bound a 2-cell in $X_{j}$; and we may suppose that $J_{i}$ is inmost in Bd $C_{j}^{\prime}$, in the sense that

$$
D_{i} \subset \mathrm{Bd} C_{j}^{\prime}-F
$$

contains no other polygon $J_{k}$ which satisfies the conditions for $J_{i}$. Thus every $J_{k}$ that lies in Int $D_{i}$ bounds a 2-cell in

$$
\text { Bd } S \cap \bigcap_{r} f^{r}\left(\text { Int } C_{j}\right) \text {. }
$$

It follows that $J_{i}$ bounds a 2-cell $D_{i}^{\prime}$, lying in $\cap_{r} f^{r}$ (Int $C_{j}$ ), such that Int $D_{i}^{\prime}$ lies in either $S_{0}-$ Int $S$ or $S-F$. $D_{i}^{\prime}$ can be chosen so as to lie in the union 
of Int $D_{j}^{-} \cup$ Int $D_{j}^{+}$and any given neighborhood of $\mathrm{Bd} S$. Therefore we may assume that

$$
D_{i}^{\prime} \cap \operatorname{Bd} C_{j}^{\prime} \subset \operatorname{Int} D_{j}^{-} \cup \operatorname{Int} D_{j}^{+}
$$

and that

$$
\bigcup_{r} f^{r}\left(D_{i}^{\prime}\right) \cap A_{j}=\varnothing .
$$

(See the proof of Lemma 6.4.1 for the "forcing off" process that is needed here.)

Now $\operatorname{Pr} \mid J_{i}$ is a loop $L$ in $\operatorname{Int} X_{j}=\operatorname{Int} \operatorname{Pr} \tilde{X}_{j}$, and $L$ is contractible in one of the sets

$$
\operatorname{Pr}\left[\left(S_{0}-\operatorname{Int} S\right) \cap \bigcap_{r} f^{r}\left(\operatorname{Int} C_{j}\right)\right], \quad \operatorname{Pr}\left[(S-F) \cap \bigcap_{r} f^{r}\left(\operatorname{Int} C_{j}\right)\right] .
$$

If $L$ were contractible in $X_{j}$, then $J_{i}$ would be contractible in $\tilde{X}_{j}$, which is false. Therefore $L$ is not contractible in $X_{j}$. We now apply Theorem 3.5 (the Loop Theorem, second form) to the 3-manifold

$$
M^{3}=\operatorname{Pr}\left[\left(\operatorname{Int} S_{0}-\operatorname{Bd} S\right) \cap \bigcap_{r} f^{r}\left(\operatorname{Int} C_{j}\right)\right] \cup \operatorname{Int} X_{j}-\operatorname{Pr} F
$$

and the 2-manifold Int $X_{j}$, which forms a closed set in the space $M^{3}$. By Theorem 3.5 it follows that there is a 2-cell $\Delta$, polyhedral relative to $K(U)$, lying in

$$
\operatorname{Pr}\left[\bigcap_{r} f^{r}\left(\operatorname{Int} C_{j}\right)\right]-\operatorname{Pr} F
$$

such that

$$
\Delta \cap \operatorname{Pr} \operatorname{Bd} S=\operatorname{Bd} \Delta \subset \operatorname{Int} X_{j},
$$

and such that $\mathrm{Bd} \Delta$ is not contractible in Int $X_{j}$, and hence not in $X_{j}$. Since $\Delta$ can be chosen in an arbitrarily small neighborhood of $\operatorname{Pr} D_{i}^{\prime}$, and $\cup_{r} f^{r}\left(D_{i}^{\prime}\right)$ $\cap A_{j}=\varnothing$, we may assume that $\Delta \cap \operatorname{Pr} A_{j}=\varnothing$. It follows that $\operatorname{Pr}^{-1} \Delta \cap A_{j}$ $=\varnothing$.

Now Bd $\Delta$ can be lifted, so as to give polygons $J_{k}^{\prime}$ such that $\operatorname{Pr} J_{k}^{\prime}=\operatorname{Bd} \Delta$. Since $\operatorname{Pr}$ is a local homeomorphism, it follows that $\operatorname{Pr}^{-1} \mathrm{Bd} \Delta$ is the union of exactly $n$ such polygons $J_{k}^{\prime}(1 \leqslant k \leqslant n)$. We choose the notation so that $J_{k+1}^{\prime}=f\left(J_{k}^{\prime}\right)$, with subscripts modulo $n$. The polygons $J_{k}^{\prime}$ cannot be latitudinal in $S$, because they bound 2-cells in $S^{3}-F$. By Theorem 6.4 it follows that each $J_{k}^{\prime}$ bounds a 2-cell $d_{k}$ in Bd $S$; and we have $d_{k+1}=f\left(d_{k}\right)$. Different sets $J_{k}^{\prime}$ are disjoint. It follows that if two different sets $d_{k}$ intersect, then one of them lies in the interior of the other, which is impossible, because $f$ is periodic. Therefore different sets $d_{k}$ are disjoint. 
Now $\operatorname{Pr}^{-1} \Delta$ is the union of exactly $n$ disjoint 2-cells $\Delta_{k}$, with

$$
\operatorname{Bd} \Delta_{k}=J_{k}^{\prime}, f\left(\Delta_{k}\right)=\Delta_{k+1} \text {. }
$$

In Bd $S$, we replace each $d_{k}$ by the corresponding $\Delta_{k}$. This gives a torus $T^{\prime}$. Evidently $T^{\prime}$ separates $F$ from Bd $S_{0}$ in $M$. Therefore, by Theorem 4.10, $T^{\prime}$ is the boundary of a solid torus $S^{\prime}$, such that $S^{\prime}$ is $f$-invariant. By arbitrarily small changes in $\Delta$, preserving all the stated properties of $\Delta$, we can produce a situation in which $\mathrm{Bd} S^{\prime}$ is in general position relative to each set $\mathrm{Bd} C_{i}^{\prime}$ $\left(C_{i}^{\prime} \in \mathbf{C}\right)$.

Now define a new set $X_{j}^{\prime}$ by deleting $X_{j} \cap \operatorname{Pr} d_{k}$ from $X_{j}$ and adding $\Delta_{k}$. Then $S^{\prime}, X_{j}^{\prime}$, and $\tilde{X}_{j}^{\prime}=\operatorname{Pr}^{-1} X_{j}^{\prime}$ satisfy all the conditions for $S, X_{j}$, and $\tilde{X}_{j}$. But all this is impossible: it preserves the stated properties of $S$, and reduces one of the numbers $p_{j}^{1}$, without increasing any of the others. This contradicts the hypothesis that $S$ was chosen so as to minimize $\Sigma_{j} p_{j}^{1}$.

7. Simplifications of $f$ and $\Omega$. Here we use the apparatus of $\S 6$.

Theorem 7.1. Let $\mathbf{B}=\left[\mathbf{P}, \mathbf{C}, \mathbf{C}^{\prime}, \mathbf{A}, S_{0}\right]$ be a barrier system for $S$, and suppose that $S$ is f-invariant. Then every set $\mathrm{Bd} S \cap C_{j}$ contains a polygon $J$ such that (1) $J$ is latitudinal and (2) $J$ is f-invariant.

Proof. By Theorem 6.3 we know that $\mathrm{Bd} S \cap \mathrm{Bd} C_{j}^{\prime}$ contains a polygon $J_{0}$ which is latitudinal in $S$. And by definition of a barrier system we have

$$
\bigcup_{r} f^{r}\left(C_{j}^{\prime}\right) \subset \bigcap_{r} f^{r}\left(\text { Int } C_{j}\right) \text {. }
$$

It follows that

$$
\bigcup_{r} f^{r}\left(J_{0}\right) \subset \operatorname{Int} C_{j} \text {. }
$$

Now $\operatorname{Pr} J_{0}$ is a polyhedron relative to $K(U)$. Let $K^{\prime}(U)$ be a subdivision of $K(U)$ in which $\operatorname{Pr} J_{0}$ forms a subcomplex, and let $N$ be a regular neighborhood of $\operatorname{Pr} J_{0}$ in $\operatorname{Pr} \operatorname{Bd} S$, defined relative to a subdivision of $K^{\prime}(U)$. Then $N$ is a 2-manifold with boundary. (We need not assert that $N$ is connected.) Therefore so also is $\tilde{N}=\operatorname{Pr}^{-1} N$; and obviously $\tilde{N}$ is $f$-invariant. We take $N$ as a sufficiently small neighborhood of $\operatorname{Pr} J_{0}$ so that $\tilde{N} \subset$ Int $C_{j}$.

Since every other set $\mathrm{Bd} S \cap C_{k}^{\prime}$ contains a latitudinal polygon, it follows as in the proof of Theorem 6.4 that every polygon in $\tilde{N}$ is latitudinal in $S$ or bounds a 2-cell in $\mathrm{Bd} S$. To $\tilde{N}$ we add all 2-cells $d \subset \mathrm{Bd} S$ such that $\mathrm{Bd} d$ is a component of $\mathrm{Bd} \tilde{N}$. This gives a 2-manifold $W$ with boundary, such that

$$
\operatorname{Bd} W \subset \operatorname{Bd} \tilde{N} \subset \operatorname{Int} C_{j} \text {, }
$$

and such that every component of $\mathrm{Bd} W$ is latitudinal in $S$. It follows that every component of $W$ is an annulus. And obviously $W$ and $\mathrm{Bd} W$ are $f$-invariant. 
Let $J_{1}$ be a latitudinal polygon in a set $\operatorname{Bd} S \cap \operatorname{Bd} C_{k}^{\prime}(k \neq j)$. Then Bd $S-J_{1}$ is homeomorphic to the interior of an annulus, and no component of $\mathrm{Bd} M$ bounds a 2-cell in $\mathrm{Bd} S-J_{1}$. It follows that there is an annulus $A \subset \operatorname{Bd} S-J_{1}$ such that $W \subset A, \operatorname{Bd} A \subset \operatorname{Bd} W$. Here $A$ is the union of $W$ and a collection of annuli $a_{i}$ whose boundaries lie in $W$ and whose interiors are disjoint from $W$. This property of $a_{i}$ is $f$-invariant. Therefore $A$ and $\mathrm{Bd} A$ are $f$-invariant. Since $f$ preserves orientation, $f$ cannot interchange the components of $\mathrm{Bd} A$. Therefore each component of $\mathrm{Bd} A$ is $f$-invariant. Since

$$
\operatorname{Bd} A \subset \operatorname{Bd} W \subset \operatorname{Bd} \tilde{N} \subset \operatorname{Int} C_{j},
$$

the theorem follows.

THEOREM 7.2. Under the hypothesis of Theorem 7.1, let $J_{j}$ be an f-invariant latitudinal polygon in $S$, lying in a set $C_{j}$. Then there is a triangulation $L(M)$ of $M$, and a homeomorphism $f^{\prime}: M \leftrightarrow M$, with fixed-point set $F^{\prime} \subset$ Int $S$, such that

(1) $F^{\prime}, S$, and $J_{j}$ form subcomplexes of $L(M)$,

(2) $F^{\prime}$ is a polygon,

(3) $F^{\prime}$ has period $n$,

(4) $f^{\prime}$ is simplicial relative to $L(M)$,

(5) $\mathrm{Cl}(M-S)$ forms a subcomplex $L^{\prime}$ of $L(M)$, and $L^{\prime}$ is a subcomplex of a subdivision of $K(\tilde{U})$,

(6) $f^{\prime}|\mathrm{Cl}(M-S)=f| \mathrm{Cl}(M-S)$, and

(7) The orbit-space $\Omega^{\prime}$ of $f^{\prime}$ is a 3-manifold, and the sets $\operatorname{Pr}^{\prime} \sigma$ (where $\sigma \in L(M)$ and $\operatorname{Pr}^{\prime}$ is the projection $M \rightarrow \Omega^{\prime}$ ) form a triangulation of $\Omega^{\prime}$.

Proof. By Theorem 7.1 we know that there is another $f$-invariant latitudinal polygon $J_{k}$ in $S$, lying in some set $C_{k}(k \neq j)$, so that $J_{j}$ and $J_{k}$ are disjoint. Thus $J_{j}$ and $J_{k}$ bound tame 2-cells $D_{j}, D_{k}$ in $S$, such that

$$
D_{p} \cap \operatorname{Bd} S=\operatorname{Bd} D_{p} \quad(p=j, k) .
$$

Since the sets Int $D_{p}$ are tame, it follows by Theorem 3.4 that we may choose them so that the sets Int $D_{p}$ are polyhedra (not necessarily finite) relative to $K(M)$. By standard methods of "cutting and pasting," we can arrange so that $D_{j} \cap D_{k}=\varnothing$.

Now $J_{j}$ and $J_{k}$ decompose $\operatorname{Bd} S$ into two annuli $a_{1}, a_{2}$. All the sets $D_{j}, D_{k}, a_{1}, a_{2}$ are tame. By Theorem 3.4, together with Lemma (2.1) of $\left[\mathbf{M}_{8}\right]$, it follows that each set $D_{j} \cup D_{k} \cup a_{q}$ is tame, and therefore bounds a 3-cell $C_{q}^{3}$. Thus

$$
S=C_{1}^{3} \cup C_{2}^{3}, \quad C_{1}^{3} \cap C_{2}^{3}=D_{j} \cup D_{k} .
$$

Let $K^{\prime}(\tilde{U})$ be a subdivision of $K(\tilde{U})$, such that $\mathrm{Bd} S$ and the sets $J_{p}$ form subcomplexes of $K^{\prime}(\tilde{U})$. Each simplex $\sigma$ of $K^{\prime}(\tilde{U})$ that lies in $\mathrm{Cl}(M-S)$ will be a simplex of $L(M)$. 
We form the rest of $L(M)$ as follows. First, we triangulate each $D_{p}$ so that the triangulation forms the join of a point $Q_{p}$ of Int $D_{p}$ and the subcomplex of $K^{\prime}(\tilde{U})$ formed by $J_{p}$. (This triangulation need not be rectilinear, or even polyhedral.) Now each set $\mathrm{Bd} C_{q}^{3}$ is already triangulated. We triangulate each $C_{q}^{3}$ in such a way that it forms the join of an interior point $R_{q}$ of $C_{q}^{3}$ with the given triangulation of $\mathrm{Bd} C_{q}^{3}$. This gives $L(M)$.

It is now easy to define $f^{\prime}$ as an extension of $f \mid \mathrm{Cl}(M-S)$. First define $f^{\prime}\left(Q_{p}\right)=Q_{p}$, and extend $f^{\prime}$ simplicially to the simplices $Q_{p} v w$ of $D_{p}$. Define $f^{\prime}\left(R_{q}\right)=R_{q}$, and extend $f^{\prime}$ simplicially to the simplices $R_{q} \sigma^{2}\left(\sigma^{2}\right.$ in $\left.\mathrm{Bd} C_{q}^{3}\right)$.

$L(M)$ and $f^{\prime}$ now satisfy (1)-(6). ( $F^{\prime}$ is the union of the four edges $Q_{p} R_{q}$ of $L(M)$.) To verify (7) we merely need to show that for each vertex $v$ of $F^{\prime}$, with closed star St $v$ in $L(M)$, the homeomorphism $f^{\prime} \mid \operatorname{St} v$ is like a periodic rotation of a round ball. This is straightforward.

8. Invariant barrier annuli. If $S$ and B satisfy all three of the conditions of Theorem 6.5, then we shall say that $S$ has no cellular oscillations in B.

TheOREM 8.1. Let B be a barrier system for $F$. Let $S_{1}$ and $S_{2}$ be f-invariant solid tori, such that $S_{2} \subset$ Int $S_{1}$; and suppose that for $i=1,2, S_{i}$ has no cellular oscillations in B. If $S_{1}$ lies in a sufficiently small neighborhood of $F$, then for each $j$ there is an annulus $A_{j}^{\prime}$ such that

(1) $A_{j}^{\prime}$ is f-invariant,

(2) $A_{j}^{\prime} \subset S_{1}-$ Int $S_{2}$,

(3) $A_{j}^{\prime} \cap\left(\mathrm{Bd} S_{1} \cup \mathrm{Bd} S_{2}\right)=\operatorname{Bd} A_{j}^{\prime}$, and this set is the union of a latitudinal polygon in $\mathrm{Bd} S_{1}$ and a latitudinal polygon in $\mathrm{Bd} S_{2}$,

(4) $\mathrm{Bd} A_{j}^{\prime} \subset$ Int $C_{j}^{\prime}$,

(5) $A_{j}^{\prime}$ intersects a set $C_{k}$ only if $k=j$ or $k=j \pm 1$, and

(6) $A_{j}^{\prime}$ is a polyhedron relative to $K(\tilde{U})$.

Proof. Take a fixed $j$, and let $d_{j}$ be as in the discussion just before the definition of a barrier system for $F$, in $\S 6$. Take $S_{1}$ in a sufficiently small neighborhood of $F$ so that

$$
\bigcup_{r} f^{r}\left(S_{1} \cap d_{j}\right) \subset \bigcap_{r} f^{r}\left(\text { Int } C_{j}^{\prime}\right) \text {. }
$$

By small changes in $d_{j}$ (preserving all conditions stated so far) we get $d_{j}$ into general position relative to Bd $S_{1}$ and Bd $S_{2}$. By hypothesis for the sets $S_{i}$ we know that every component $J$ of $d_{j} \cap \operatorname{Bd} S_{i}(i=1,2)$ is latitudinal in $S_{i}$ or bounds a 2-cell in $\mathrm{Bd} S_{i}$; and in the latter case $J$ bounds a 2-cell in Bd $S_{i} \cap \cap_{r} f^{r}\left(\operatorname{Int} C_{j}\right)$.

Lemma 8.1.1. For $i=1,2$ let $M_{i}^{2}$ be the component of $d_{j} \cap S_{i}$ that contains $P_{j}=d_{j} \cap F$. Then exactly one component $J_{i}$ of $\mathrm{Bd} M_{i}^{2}$ is latitudinal in $S_{i}$; the other components of $\mathrm{Bd} M_{i}^{2}$ bound 2-cells in $\mathrm{Bd} S_{i}$; and $J_{2}$ separates $P_{j}$ from $J_{1}$ in $d_{j}$. 
Proof. By general position, $M_{i}^{2}$ is a 2-manifold with boundary. Let $J_{i}$ be the frontier (in $d_{j}$ ) of the component of $d_{j}-M_{i}^{2}$ that contains $\mathrm{Bd} d_{j}$. Then $J_{i}$ is a polygon, because $M_{i}^{2}$ is connected. Every other component $J$ of $\mathrm{Bd} M_{i}^{2}$ bounds a 2-cell in $d_{j}-P_{j}$, and so cannot be latitudinal in $S_{i}$. Therefore each such $J$ bounds a 2-cell in Bd $S_{i}$. It follows, by a standard "forcing off" process, that $J_{i}$ is latitudinal in $S_{i}$. Since $M_{2}^{2} \subset$ Int $M_{1}^{2}, J_{2}$ lies in the interior of the 2-cell in $d_{j}$ bounded by $J_{1}$, and the lemma follows.

Note that $J_{1}$ is the inmost (in $d_{j}$ ) of the polygons in $d_{j} \cap \mathrm{Bd} S_{1}$ that are latitudinal in $S_{1}$, and $J_{2}$ is the outermost (in $d_{j}$ ) of the latitudinal polygons in $d_{j} \cap \mathrm{Bd} S_{2}$ that lie in the 2-cell in $d_{j}$ bounded by $J_{1}$.

LEMMA 8.1.2. For $i=1,2$ there is an f-invariant polyhedral annulus $a_{i}$ in Bd $S_{i}$ such that

$$
J_{i} \subset \operatorname{Int} a_{i}, \quad \operatorname{Bd} a_{i} \subset \bigcap_{r} f^{r}\left(\text { Int } C_{j}^{\prime}\right), \quad a_{i} \subset \bigcap_{r} f^{r}\left(\text { Int } C_{j}\right) .
$$

Proof of lemma. Consider the 1-dimensional polyhedron

$$
\operatorname{Pr} J_{i} \subset \operatorname{Pr} \operatorname{Bd} S_{i} \text {. }
$$

Let $N$ be a small regular neighborhood of $\operatorname{Pr} J_{i}$ in $\operatorname{Pr} \operatorname{Bd} S_{i}$, and let $\tilde{N}$ be the component of $\operatorname{Pr}^{-1} N$ that contains $J_{i}$. Then $\tilde{N}$ is a connected polyhedral 2-manifold with boundary in Bd $S_{i}$, and $J_{i} \subset$ Int $\tilde{N}$. Since

$$
\bigcup_{r} f^{r}\left(J_{1}\right) \subset \bigcap_{r} f^{r}\left(\text { Int } C_{j}^{\prime}\right),
$$

we can choose $N$ so that $\tilde{N} \subset \bigcap_{r} f^{r}$ (Int $C_{j}^{\prime}$ ). If $J$ is a component of $\mathrm{Bd} \tilde{N}$ which bounds a 2-cell $D_{J} \subset \operatorname{Bd} S_{i}$, then $D_{J} \subset \cap_{r} f^{r}$ (Int $C_{j}$ ). We add all such 2-cells to $\tilde{N}$, getting an $f$-invariant 2 -manifold $a_{i}$ with boundary, such that $J_{i} \subset$ Int $a_{i}$ and such that no component of $\mathrm{Bd} a_{i}$ bounds a 2-cell in Bd $S_{i}$. It follows that each component of $\operatorname{Bd} a_{i}$ is latitudinal in $S_{i}$. Therefore $\operatorname{Bd} a_{i}$ has exactly two components, and $a_{i}$ is an annulus, satisfying all the conditions of the lemma. (Hereafter, the notations $N, \tilde{N}$ will not refer to the above proof.)

Now $J_{1} \cup J_{2}$ is the boundary of an annulus $B_{1} \subset d_{j}-P_{j}$. The intersections Int $B_{1} \cap \mathrm{Bd} S_{i}$ may not be empty, but each of them is a finite union of disjoint polygons, each of which bounds a 2-cell in $\mathrm{Bd} S_{i}$, and hence in

$$
\text { Bd } S_{i} \cap \bigcap_{r} f^{r}\left(\text { Int } C_{j}\right) \text {. }
$$

By a standard "forcing off" process, we get an annulus $B_{2}$, polyhedral relative to $K(\tilde{U})$, such that

$$
\begin{gathered}
B_{2} \subset \bigcap_{r} f^{r}\left(\text { Int } C_{j}\right), \quad \operatorname{Bd} B_{2}=J_{1} \cup J_{2} \subset \text { Int } a_{1} \cup \operatorname{Int} a_{2}, \\
\text { Int } B_{2} \subset \text { Int } S_{1}-S_{2} .
\end{gathered}
$$

Adding to $B_{2}$ a 2-cell in $S_{2}$, with $J_{2}$ as a boundary, and with its interior in 
Int $S_{2}$, we get a 2-cell $D \subset S_{1}$, with

$$
\text { Int } D \subset \text { Int } S_{1}, \quad D \cap \operatorname{Bd} S_{i} \subset \text { Int } a_{i} \subset \bigcap_{r} f^{r}\left(\text { Int } C_{j}\right) \quad(i=1,2) \text {, }
$$

and

$$
D-\operatorname{Int} S_{2} \subset \bigcap_{r} f^{r}\left(\text { Int } C_{j}\right)
$$

so that

$$
\operatorname{Pr} D-\operatorname{Pr} \text { Int } S_{2} \subset \operatorname{Pr} \bigcap_{r} f^{r}\left(\text { Int } C_{j}\right) \text {. }
$$

We now use $S_{2}$ as the $S$ of the hypothesis of Theorem 7.2. Consider the $f^{\prime}, \Omega^{\prime}, \operatorname{Pr}^{\prime}: M \rightarrow \Omega^{\prime}$ given by the conclusion of Theorem 7.2. Thus $\operatorname{Pr}^{\prime} S_{2}$ is a PL 3-manifold with boundary, and so also is $\operatorname{Pr}^{\prime} S_{1}$. Note that $\operatorname{Pr}^{\prime}(M-$ Int $\left.S_{2}\right)=\operatorname{Pr}\left(M-\right.$ Int $\left.S_{2}\right)$; in fact, $\operatorname{Pr}^{\prime} \mid\left(M-\right.$ Int $\left.S_{2}\right)=\operatorname{Pr} \mid\left(M-\right.$ Int $\left.S_{2}\right)$. Let $W$ be a closed neighborhood of $\operatorname{Pr}\left(D \cup \operatorname{Int} a_{1} \cup \operatorname{Int} a_{2}\right)$, in $\operatorname{Pr}^{\prime}\left(S_{1}\right)$ regarded as a space, such that

(1) $W$ is a polyhedral 3-manifold with boundary,

(2) $W \cap \operatorname{Pr}^{\prime} \operatorname{Bd} S_{i}=\operatorname{Pr} a_{i}(i=1,2)$, and

(3) $W-\operatorname{Pr}$ Int $S_{2} \subset \operatorname{Pr} \cap{ }_{r} f^{r}\left(\right.$ Int $\left.C_{j}\right)$.

Let $N=\operatorname{Pr} a_{1}$, and let $M^{3}=$ Int $W \cup$ Int $N$. Then $M^{3}$ is a 3-manifold with boundary, and $\mathrm{Bd} M^{3}=$ Int $N$. Evidently there is a loop in Int $N$ (namely, $\operatorname{Pr}\left(J_{1}\right)$ which is contractible in $M^{3}$ but not in Int $N$. By the Loop Theorem it follows that there is a PL 2-cell $\Delta_{1}$ in $M^{3}$, with

$$
\operatorname{Bd} \Delta_{1}=\Delta_{1} \cup \operatorname{Int} N \text {, }
$$

such that $\mathrm{Bd} \Delta_{1}$ is not contractible in Int $N$. By an isotopy we can move $\Delta_{1}$ so as to get a PL 2-cell $\Delta$ such that

$$
\Delta \subset W, \quad \operatorname{Bd} \Delta=\Delta \cap \operatorname{Bd} W \subset \operatorname{Bd} N,
$$

so that $\mathrm{Bd} \Delta$ is not contractible in $N$.

If $\mathrm{Bd} \Delta$ were contractible in $\operatorname{Pr}^{\prime} \mathrm{Bd} S_{1}$, then each component of the annulus $N=\operatorname{Pr}^{\prime} a_{1}$ would be contractible in $\operatorname{Pr}^{\prime} \operatorname{Bd} S_{1}$; each component of $\operatorname{Bd} a_{1}$ would be contractible in $\mathrm{Bd} S_{1}$, and so $J_{1}$ would be contractible in Bd $S_{1}$, which is false. Thus we have the following:

(i) $\Delta \cap \operatorname{Bd} \operatorname{Pr}^{\prime} S_{1}=\operatorname{Bd} \Delta \subset \operatorname{Bd}^{\operatorname{Pr}^{\prime}} a_{1}$,

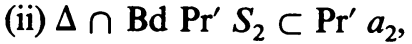

(iii) Int $\Delta \subset \operatorname{Pr}^{\prime}$ Int $S_{1}$,

(iv) $\mathrm{Bd} \Delta$ is not contractible in $\operatorname{Pr}^{\prime} \mathrm{Bd} S_{1}$, and

(v) $\left(\Delta-\operatorname{Pr}^{\prime} \operatorname{Int} S_{2}\right)-\operatorname{Pr}^{\prime} \cap_{r} f^{r}\left(\operatorname{Int} C_{j}\right)$ lies in a finite union of disjoint polyhedral 2-cells, disjoint from $\operatorname{Pr}^{\prime} B d S_{1}$, from $\operatorname{Pr}^{\prime} B d S_{2}$, and from one another.

(In fact, at the outset we have

$\left(\mathrm{v}^{\prime}\right) \Delta-\operatorname{Pr}^{\prime}$ Int $S_{2} \subset \operatorname{Pr}^{\prime} \cap{ }_{r} f^{r}\left(\operatorname{Int} C_{j}\right)$. 
But (v) needs to be stated in such a form that it would be preserved by certain hypothetical operations to be discussed presently.)

We may also assume that

(vi) $\Delta$ is in general position relative to $\operatorname{Pr}^{\prime} \mathrm{Bd}_{2}$, and $\operatorname{Pr}^{-1}$ Int $\Delta$ is in general position relative to each set $\mathrm{Bd} C_{k}^{\prime}$.

(Note that (i) implies that $\mathrm{Bd} \Delta \cap \operatorname{Bd} C_{k}^{\prime}=\varnothing$ for each $k$.)

Finally, we may assume that

(vii) Subject to conditions (i)-(vi), $\Delta$ is chosen so as to minimize the number $p$ of components of $\Delta \cap \operatorname{Pr}^{\prime} \operatorname{Bd} S_{2}$.

LemMa 8.1.3. No component $J$ of $\Delta \cap \operatorname{Pr}^{\prime} B d S_{2}$ bounds a 2-cell in $\operatorname{Pr}^{\prime} \operatorname{Bd} S_{2}$.

Proof of Lemma. Suppose that

$$
J=\operatorname{Bd} D_{J}, \quad D_{J} \subset \operatorname{Pr}^{\prime} \operatorname{Bd} S_{2} .
$$

We may suppose that $J$ is inmost in $\operatorname{Pr}^{\prime} \operatorname{Bd} S_{2}$, in the sense that Int $D_{J}$ contains no component of $\Delta \cap \operatorname{Pr}^{\prime} \operatorname{Bd} S_{2}$. Now $J$ bounds a 2-cell $D_{J}^{\prime} \subset \Delta$. We substitute $D_{J}$ for $D_{J}^{\prime}$ in $\Delta$, and force the resulting 2-cell slightly off $\operatorname{Pr}^{\prime} \operatorname{Bd} S_{2}$ in the neighborhood of $D_{J}$. Minor adjustments restore condition (vi). All this is impossible, because it preserves (i)-(vi) and reduces the $p$ of (vii).

Now let $D^{\prime}$ be a 2-cell which is a lifting of $\Delta$, so that $\operatorname{Pr}^{\prime} D^{\prime}=\Delta$. Let $A_{j}^{\prime}$ be the component of $D^{\prime}-\operatorname{Int} S_{2}$ that contains $\operatorname{Bd} D^{\prime}$.

LEMMA 8.1.4. Every component of $\mathrm{Bd} A_{j}^{\prime}-\mathrm{Bd} S_{1}$ is latitudinal in $S_{2}$.

Proof of LEMma. Since each such component $J$ is disjoint from a latitudinal polygon (lying in some set $\mathrm{Bd} S_{2} \cap \operatorname{Bd} C_{r}^{\prime}(r \neq j)$ ), it follows that $J$ is latitudinal in $S_{2}$ or bounds a 2-cell in $\mathrm{Bd}_{2}$. If the latter held, then $\operatorname{Pr}^{\prime} J$ would bound a 2-cell in $\operatorname{Pr}^{\prime} \mathrm{Bd} S_{2}$, which is false.

LEMMA 8.1.5. Every component of $\mathrm{Bd} A_{j}^{\prime}-\mathrm{Bd} S_{1}$ is f-invariant. So also is $A_{j}^{\prime}$.

Proof of lemMa. Evidently the entire set $\operatorname{Pr}^{\prime-1} \Delta$ is $f^{\prime}$-invariant. Applying Theorem 7.1 in some set $C_{r}^{\prime}(r \neq j)$, we express $\mathrm{Bd} S_{2}$ as the union of two $f$-invariant annuli, whose intersection is the boundary of each, such that Bd $S_{2} \cap \operatorname{Pr}^{\prime-1} \Delta$ lies in the union of their interiors. Since $f$ is periodic, and preserves orientation, the components of $\mathrm{Bd} A_{j}^{\prime} \cap \mathrm{Bd} S_{2}$ cannot be permuted by $f^{\prime}$ (which agrees with $f$ except in Int $S_{2}$ ). Therefore each of these components is $f$-invariant. Therefore so also is $A_{j}^{\prime}$.

LEMMA 8.1.6. $A_{j}^{\prime}$ is an annulus.

Proof of Lemma. We need to show that $\mathrm{Bd} A_{j}^{\prime} \cap \mathrm{Bd} S_{2}$ is connected. Now $A_{j}^{\prime}$ can be regarded as a complex (that is, a subcomplex of a subdivision of $K(\tilde{U})$ ). Assign orientations to the simplices of $A_{j}^{\prime}$, in such a way that by 
assigning coefficient 1 to each 2-simplex of $A_{j}^{\prime}$ we get a 2 -chain $C^{2}$, with integers as coefficients, such that

$$
\partial C^{2}=Z^{1}=Z_{1}^{1}+Z_{2}^{1},
$$

where $Z_{i}^{1}$ is a 1 -cycle on $\mathrm{Bd} S_{i}(i=1,2)$. Now

$$
Z_{2}^{1}=\sum_{s} Y_{s}^{1}
$$

where each $Y_{s}^{1}$ has constant coefficient 1 or -1 on its polygonal carrier $\left|Y_{s}^{1}\right|$, which is a component of $A_{j}^{\prime} \cap \mathrm{Bd} S_{2}$.

Suppose that there is more than one such component $\left|Y_{s}^{1}\right|$. Since all of them are latitudinal in $S_{2}$, we have $Y_{r}^{1} \sim \pm Y_{s}^{1}$ for every $r$ and $s$. Therefore there are two cycles $Y_{r}^{1}$, say, $Y_{1}^{1}$ and $Y_{2}^{1}$, such that $Y_{1}^{1}+Y_{2}^{1} \sim 0$ on $\operatorname{Bd} S_{2}$. Let $b$ be a broken line in $A_{j}^{\prime}$, from a point of $\left|Y_{1}^{1}\right|$ to a point of $\left|Y_{2}^{1}\right|$, intersecting $\mathrm{Bd} A_{j}^{\prime}$ only at its end-points, and let $N$ be a small regular neighborhood of $\left|Y_{1}^{1}\right| \cup\left|Y_{2}^{1}\right| \cup b$ in $A_{j}^{\prime}$. Then $\mathrm{Bd} N$ is a polygon $J$, and $J$ carries a 1-cycle $Z_{J}$ such that $Z_{J} \sim Y_{1}^{1}+Y_{2}^{1} \sim 0$ on $S_{1}-$ Int $S_{2}$. Therefore $Z_{J} \sim 0$ on Int $S_{1}-$ Int $S_{2}$. Evidently $J$ is not contractible in $A_{j}^{\prime}: J$ separates $A_{j}^{\prime}$ into two connected sets each of which contains a component of $\mathrm{Bd} A_{j}^{\prime}$.

By Theorem 4.10 we know that Int $S_{1}-S_{2}$ is the interior of a toroidal shell, and

$$
\pi\left(\operatorname{Int} S_{1}-S_{2}\right) \approx H_{1}\left(\text { Int } S_{1}-S_{2}, \mathbf{Z}\right) \approx \mathbf{Z}+\mathbf{Z} .
$$

Thus the canonical homomorphism

$$
\pi\left(\text { Int } S_{1}-S_{2}\right) \rightarrow H_{1}\left(\text { Int } S_{1}-S_{2}, \mathrm{Z}\right)
$$

is an isomorphism. Since $Z_{J} \sim 0$ on Int $S_{1}-S_{2}$, it follows that any loop $L$ : $S^{1} \rightarrow J$ that traverses $J$ exactly once is contractible in Int $S_{1}-S_{2}$. Since the homomorphism

$$
\left(\operatorname{Pr} \mid \operatorname{Int} A_{j}^{\prime}\right)^{*}: \pi\left(A_{j}^{\prime}\right) \rightarrow \pi\left(\operatorname{Pr} A_{j}^{\prime}\right)
$$

is injective, it follows that $\operatorname{Pr} L: S^{1} \rightarrow \operatorname{Pr} \operatorname{Int} A_{j}^{\prime}$ is not contractible in Pr Int $A_{j}^{\prime}$. We now apply the Loop Theorem (second form) to the 3-manifold $\operatorname{Pr}\left(\operatorname{Int} S_{1}-S_{2}\right)$ and the 2-manifold $\operatorname{Pr}$ Int $A_{j}^{\prime}$. This gives a PL 2-cell

$$
\Delta_{1} \subset \operatorname{Pr}\left(\operatorname{Int} S_{1}-S_{2}\right) \text {, }
$$

with

$$
\Delta_{1} \cap \operatorname{Pr} A_{j}^{\prime}=\operatorname{Bd} \Delta_{1} \subset \operatorname{Pr} \operatorname{Int} A_{j}^{\prime},
$$

such that $\operatorname{Bd} \Delta_{1}$ is not contractible in $\operatorname{Pr} A_{j}^{\prime}$. Obviously $\Delta_{1}$ can be slid off all the 2-cells mentioned in (v) above, and so we may assume that

$$
\operatorname{Bd} \Delta_{1} \subset \operatorname{Pr} \bigcap_{r} f^{r}\left(\operatorname{Int} C_{j}\right) \text {. }
$$

Now Bd $\Delta_{1}$ bounds a 2-cell $\Delta_{2}$ in $\Delta$, and Int $\Delta_{2}$ must contain at least one 
component of $\Delta \cap \operatorname{Pr} \operatorname{Bd} S_{2}$. We replace $\Delta_{2}$ by $\Delta_{1}$ in $\Delta$, getting a 2-cell $\Delta^{\prime}$. We then move Int $\Delta^{\prime}$ slightly, so that $\operatorname{Pr}^{-1}$ Int $\Delta^{\prime}$ is in general position relative to each set $\mathrm{Bd} C_{j}^{\prime}$.

But all this is impossible: it preserves conditions (i) $-(v)$, and reduces the number of components of $\Delta \cap \operatorname{Pr}^{\prime} \operatorname{Bd} S_{2}$. The lemma follows.

Thus $A_{j}^{\prime}$ is an annulus. By conditions (i) and (ii) for $\Delta$ we have

$$
\operatorname{Pr}^{\prime} \operatorname{Bd} A_{j}^{\prime} \subset \operatorname{Pr}^{\prime} \operatorname{Bd} a_{1} \cup \operatorname{Pr}^{\prime} a_{2} \text {. }
$$

Now $A_{j}^{\prime}$ satisfies (1)-(3) and (6) of Theorem 8.1. To get an $A_{j}^{\prime}$ satisfying (4), we move the polygon $\Delta \cap \operatorname{Pr}^{\prime} \operatorname{Bd} S_{2}$ onto a component of $\operatorname{Pr}^{\prime} \operatorname{Bd} a_{2}$, by an isotopy $\Omega^{\prime} \leftrightarrow \Omega^{\prime}, \operatorname{Pr}^{\prime} \operatorname{Bd} S_{2} \leftrightarrow \operatorname{Pr}^{\prime} \operatorname{Bd} S_{2}$ which differs from the identity only in a small neighborhood of $\operatorname{Pr}^{\prime} a_{2}$. This gives a new $\Delta$, satisfying (i)-(iv). Since $a_{2} \subset \cap_{r} f^{r}\left(\right.$ Int $\left.C_{j}\right)$, (v) is also satisfied. The new $\Delta$ can be moved into general position so as to satisfy (vi). Lifting, we get a new $A_{j}^{\prime}$ such that

$$
\operatorname{Bd} A_{j}^{\prime} \subset \operatorname{Bd} a_{1} \cup \operatorname{Bd} a_{2} \subset \operatorname{Int} C_{j}^{\prime} \text {. }
$$

Thus the new $A_{j}^{\prime}$ satisfies (1)-(4) and (6).

It remains only to show that $A_{j}^{\prime}$ can be chosen so as to satisfy (5). The proof is very similar to that of Theorem 6.5 , as follows. Consider the sets

$$
A_{j}^{\prime} \cap \operatorname{Bd} C_{j-1}^{\prime}, \quad A_{j}^{\prime} \cap \mathrm{Bd} C_{j+1}^{\prime} .
$$

Each of these is a finite union of disjoint polygons $J$. If each of these bounds a 2-cell in $A_{j}^{\prime} \cap \cap_{r} f^{r}\left(\right.$ Int $\left.C_{j-1}\right)$ or $A_{j}^{\prime} \cap \cap_{r} f^{r}\left(\right.$ Int $\left.C_{j+1}\right)$, then $A_{j}^{\prime}$ satisfies (5). Thus, if (5) does not hold, then there is such a $J$, lying, say, in $A_{j}^{\prime} \cap \mathrm{Bd} C_{j-1}^{\prime}$, such that $J$ does not bound a 2-cell in $A_{j}^{\prime} \cap \cap_{r} f^{r}\left(\right.$ Int $\left.C_{j-1}\right)$.

By (v), each such $J$ bounds a 2-cell in $A_{j}^{\prime}$. Now $J$ bounds a 2-cell $D_{J}$ in Bd $C_{j-1}^{\prime}-A_{j-1}$. If $D_{J}$ intersects $F$, then every polygon in $\mathrm{Bd} S_{1} \cap \mathrm{Bd} C_{j-1}^{\prime}$ bounds a 2-cell in $S_{1}$, disjoint from $F$; and this is impossible, because some polygon in $\mathrm{Bd} S_{1} \cap \mathrm{Bd} C_{j-1}^{\prime}$ is latitudinal in $S_{1}$. Therefore

$$
D_{J} \subset\left(\operatorname{Bd} C_{j-1}^{\prime}-A_{j-1}\right)-F .
$$

We may suppose that $D_{J}$ is irreducible with respect to its stated properties, so that if $J^{\prime}$ is a polygon in $A_{j}^{\prime} \cap$ Int $D_{J}$, then $J^{\prime}$ bounds a 2-cell in $A_{j}^{\prime} \cap$ $\cap_{r} f^{r}\left(\right.$ Int $\left.C_{j-1}\right)$. No component $J^{\prime}$ of

$$
\left(\mathrm{Bd} S_{1} \cup \mathrm{Bd} S_{2}\right) \cap \mathrm{Bd} C_{j-1}^{\prime}
$$

that lies in $D_{J}$ can be latitudinal in $S_{1}$ or $S_{2}$; and by Theorem 6.4 it follows that every such $J^{\prime}$ bounds a 2-cell in $\mathrm{Bd} S_{1}$ (or Bd $S_{2}$ ). By the hypothesis of Theorem 8.1., $J^{\prime}$ bounds a 2-cell in

$$
\text { Bd } S_{i} \cap \bigcap_{r} f^{r}\left(\text { Int } C_{j-1}\right) \quad(i=1 \text { or } i=2) \text {. }
$$

By the usual forcing off process we conclude that $J$ bounds a 2-cell $D_{J}^{\prime}$ in 


$$
\left(\text { Int } S_{1}-S_{2}\right) \cap \bigcap_{r} f^{r}\left(\operatorname{Int} C_{j-1}\right)
$$

such that $D_{j}^{\prime} \cap A_{j}^{\prime}=J$.

All this can be ruled out by a minimality assumption similar to the one used in the proof of Theorem 6.5. For each $j$, consider the set

$$
Y=\operatorname{Pr}\left[A_{j}^{\prime} \cap \bigcup_{r} f^{r}\left(C_{j-1}^{\prime}\right)\right] .
$$

This is a finite polyhedron (relative to $K(U)$ ). Let $X_{j-1}$ be a polyhedral 2-manifold with boundary which forms a neighborhood of $Y$ in $\operatorname{Pr} A_{j}^{\prime}$, such that

$$
X_{j-1} \subset \operatorname{Pr} \bigcap_{r} f^{r}\left(\operatorname{Int} C_{j-1}\right) \text {. }
$$

Then $X_{j-1}$ and $\tilde{X}_{j-1}=\operatorname{Pr}^{-1} X_{j-1}$ are 2-manifolds with boundary. Let $H_{1}\left(X_{j-1}\right)$ be the 1-dimensional homology group of $X_{j-1}$, with coefficients in the additive group $\mathbf{Z}_{2}$ of integers modulo 2 .

Let $J$ and $D_{J}^{\prime}$ be as in the preceding discussion. Let $V$ be the component of

$$
\left[\left(\operatorname{Int} S_{1}-S_{2}\right) \cap \bigcap_{r} f^{r}\left(\operatorname{Int} C_{j-1}\right)\right]-A_{j}^{\prime}
$$

that contains Int $D_{J}^{\prime}$. Since Int $S_{1}, S_{2}, \cap_{r} f^{r}\left(\right.$ Int $\left.C_{j-1}\right)$, and $A_{j}^{\prime}$ are all $f$ invariant, so also is $V$. Let

$$
W=\operatorname{Pr} V \cup \operatorname{Int} X_{j-1} .
$$

Then $W$ is a $\operatorname{PL} 3$-manifold with boundary, with $\mathrm{Bd} W=\operatorname{Int} X_{j-1}$, and $\operatorname{Pr} \mid J$ is a loop in $\mathrm{Bd} W$, contractible in $W$ but not in $\mathrm{Bd} W$. By the Loop Theorem it follows that there is a polyhedral 2-cell $\Delta \subset W$ such that $B d \Delta=\Delta \cap$ $\mathrm{Bd} W$ and $\mathrm{Bd} \Delta$ is not contractible in $\mathrm{Bd} W$. As in the proof of Theorem 6.5, Bd $\Delta$ can be lifted, to give polygons $J_{k}^{\prime}(1<k<n)$ in $A_{j}^{\prime}$, such that $\operatorname{Pr} J_{k}^{\prime}=$ $\mathrm{Bd} \Delta$. As before, these polygons are disjoint, and bound disjoint 2-cells $d_{k}$ in $A_{j}^{\prime}$. Similarly, $\operatorname{Pr}^{-1} \Delta$ is the union of $n$ disjoint 2-cells $\Delta_{k}$, with $\operatorname{Bd} \Delta_{k}=J_{k}^{\prime}$; and since $\Delta \subset W$ we have

$$
\Delta_{k} \subset\left(\text { Int } S_{1}-S_{2}\right) \cap \bigcap_{r} f^{r}\left(\text { Int } C_{j-1}\right) .
$$

In $A_{j}^{\prime}$, we replace each $d_{k}$ by the corresponding $\Delta_{k}$, thus getting an $f$-invariant annulus $A_{j}^{\prime \prime}$. By arbitrarily small changes in $\Delta$, we can arrange so that $A_{j}^{\prime \prime}$ is in general position relative to $\mathrm{Bd} C_{j-1}^{\prime}$.

Now define a new set $X_{j-1}^{\prime}$ by deleting $X_{j-1} \cap \operatorname{Pr} d_{k}$ from $X_{j-1}$ and replacing it with $\Delta$. Then $A_{j}^{\prime \prime}$ and $X_{j-1}^{\prime}$ have all the stated properties of $A_{j}^{\prime}$ and $X_{j-i}: A_{j}^{\prime \prime}$ is an $f$-invariant annulus, satisfying (1)-(4) and (6) of Theorem 8.1; $X_{j-1}^{\prime}$ is a polyhedral 2-manifold with boundary, forming a neighborhood of 


$$
Y^{\prime}=\operatorname{Pr}\left[A_{j}^{\prime \prime} \cap \bigcup_{r} f^{r}\left(C_{j-1}^{\prime}\right)\right]
$$

in $\operatorname{Pr} A_{j}^{\prime \prime}$, and

$$
X_{j-1}^{\prime} \subset \operatorname{Pr} \bigcap_{r} f^{r}\left(\operatorname{Int} C_{j-1}\right)
$$

But the operation $A_{j}^{\prime} \rightarrow A_{j}^{\prime \prime}, X_{j-1} \rightarrow X_{j-1}^{\prime}$ reduces the dimension of $H_{1}\left(X_{j-1}\right)$ (as a vector space over $\mathbf{Z}_{2}$ ), without increasing the dimension of the similarly defined group $H_{1}\left(X_{j+1}\right)$. Therefore, to get an $A_{j}^{\prime}$ satisfying (1)-(6), it is sufficient to choose an $A_{j}^{\prime}$ satisfying (1)-(4) and (6), in such a way as to minimize the sum of the dimensions of $H_{1}\left(X_{j-1}\right)$ and $H_{1}\left(X_{j+1}\right)$.

\section{Eliminating annular oscillations.}

Theorem 9.1. Let $\mathbf{B}^{\prime}=\left[\mathbf{P}, \mathbf{C}, \mathbf{C}^{\prime}, \mathbf{A}, S_{0}\right]$ be a barrier system for $F$, and suppose that the number of points in $P$ is $4 m(m \geqslant 3)$. Let $\mathbf{B}$ be the barrier system which uses the same $S_{0}$, and uses the points $P_{4 j}$ and the sets $C_{4 j}, C_{4 j}^{\prime}, A_{4 j}$. Then every neighborhood of $F$ contains an f-invariant solid torus $S$ such that

(1) $S$ and $\mathbf{B}$ satisfy all the conditions for $S_{1}$ and $\mathbf{B}$ in Theorem 8.1, and

(2) $\mathrm{Bd} S$ is the union of a collection of annuli $M_{j}(1<j<m)$, intersecting only on their boundaries, such that (2.1) $M_{j}$ is f-invariant, (2.2) $\mathrm{Bd} M_{j}$ is the union of a latitudinal polygon in $S \cap \operatorname{Int} C_{4 j}$ and a latitudinal polygon in $S \cap \operatorname{Int} C_{4 j+4}$, and (2.3) $M_{j}$ intersects $C_{4 k}$ only if $k=j$ or $k=j+1$.

Proof. Let $S_{1}$ be a solid torus such that $S_{1}$ and $\mathbf{B}^{\prime}$ satisfy all the conditions for $S_{1}$ and B in Theorem 8.1. Thus if $S_{2}$ is a solid torus lying in Int $S_{1}$, and $S_{2}$ has no cellular oscillations in $\mathbf{B}^{\prime}$, then the conclusion of Theorem 8.1 holds for $S_{1}, S_{2}$, and $\mathbf{B}^{\prime}$. In Theorem 8.1 it was merely required that $S_{1}$ be a "sufficiently small" neighborhood of $F$, with no cellular oscillations in $\mathbf{B}$. Thus $S_{2}$ and $\mathbf{B}^{\prime}$ automatically satisfy the conditions for $S_{1}$ and $\mathbf{B}$ in Theorem 8.1.

Now $F$ intersects each set $\mathrm{Bd} C_{4 j+2}^{\prime}$ in two points $P_{4 j+2}^{-}, P_{4 j+2}^{+}$, and these points have 2-cell neighborhoods $d_{4 j+2}^{-}, d_{4 j+2}^{+}$in $\mathrm{Bd} C_{4 j+2}^{\prime}$, disjoint from Bd $S_{1}$. By Theorem 6.5 there s a solid torus $S$ such that (i) $S$ has no cellular oscillations in $\mathbf{B}^{\prime}$, (ii) $S \subset$ Int $S_{1}$, and (iii) for each $j$,

$$
S \cap \operatorname{Bd} C_{4 j+2}^{\prime} \subset \text { Int } d_{4 j+2}^{-} \cup \text { Int } d_{4 j+2}^{+} \text {. }
$$

Now $P_{4 j+2}^{-}$and $P_{4 j+2}^{+}$have 2-cell neighborhoods

$$
d_{4 j+2}^{\prime-} \subset \text { Int } d_{4 j+2}^{-}, \quad d_{4 j+2}^{\prime+} \subset \text { Int } d_{4 j+2}^{+}
$$

in Bd $C_{4 j+2}^{\prime}$, disjoint from Bd $S$. Let $S_{2}$ be an $f$-invariant solid torus as in Theorem 8.1, such that

$$
S_{2} \cap \operatorname{Bd~} C_{4 j+2}^{\prime} \subset \text { Int } d_{4 j+2}^{\prime+} \cup \text { Int } d_{4 j+2}^{\prime-} ;
$$


and let $\left\{A_{4 j+2}^{\prime}\right\}$ be as in the conclusion of Theorem 8.1. (Throughout, we are using $\mathbf{B}^{\prime}$ as the $\mathbf{B}$ of Theorem 8.1, but using only the resulting annuli of the form $A_{4 j+2}^{\prime}$.) From (5) of Theorem 8.1 it follows that every two different sets $A_{4 j+2}^{\prime}$ are disjoint, and that none of them intersects any set $C_{4 k}$.

By Theorem 8.1, we have Bd $A_{4 j+2}^{\prime} \subset$ Int $C_{4 j+2}^{\prime}$. The polygons in $\mathrm{Bd} S_{1} \cap$ Bd $C_{4 j+2}^{\prime}$ which are latitudinal in $S_{1}$ decompose Bd $S_{1}$ into a finite collection of annuli, one of which, $A_{1, j}$, contains

$$
\text { Bd } A_{4 j+2}^{\prime} \cap \operatorname{Bd} S_{1} \subset \operatorname{Int} C_{4 j+2}^{\prime}
$$

in its interior. Similarly, the latitudinal polygons in $\mathrm{Bd} S_{2} \cap \mathrm{Bd} C_{4 j+2}^{\prime}$ decompose $\mathrm{Bd} S_{2}$ into annuli, one of which, $A_{2, j}$, contains the other component of $\mathrm{Bd} A_{4 j+2}^{\prime}$ in its interior. The components $J$ of $\mathrm{Bd} S_{i} \cap$ Bd $C_{4 j+2}^{\prime}$ which are not latitudinal in $S_{i}$ bound 2-cells in Bd $S_{i}$ (Theorem 6.4). Since $S_{1}$ and $S_{2}$ have no cellular oscillations in $\mathbf{B}^{\prime}$, it follows that

$$
A_{i j} \subset \bigcap_{r} f^{r}\left(\operatorname{Int} C_{4 j+2}\right) \quad(i=1,2) \text {. }
$$

We now form the union $L_{j}$ of

(1) The annulus $A_{4 j+2}$ (as in the definition of a barrier system, just before Theorem 6.1),

(2) An annulus in $A_{1, j}$, bounded by the union of $\mathrm{Bd} A_{4 j+2}^{\prime} \cap \mathrm{Bd} S_{1}$ and a latitudinal polygon $J_{1} \subset \mathrm{Bd} S_{1} \cap \mathrm{Bd} C_{4 j+2}^{\prime}$,

(3) $A_{4 j+2}^{\prime}$,

(4) An annulus in $A_{2, j}$, bounded by the union of $\mathrm{Bd} A_{4 j+2}^{\prime} \cap \mathrm{Bd} S_{2}$ and a latitudinal polygon $J_{2}$ in $\mathrm{Bd} S_{2} \cap \mathrm{Bd} C_{4 j+2}^{\prime}$,

(5) An annulus in $\mathrm{Bd} C_{4 j+2}^{\prime}-\left(d_{4 j+2}^{-} \cup d_{4 j+2}^{+}\right)$, bounded by $J_{1}$ and a boundary component of $A_{4 j+2}$, and

(6) A 2-cell in $d_{4 j+2}^{\prime+}$ or $d_{4 j+2}^{\prime-}$, bounded by $J_{2}$.

LEMMA 9.1.1. $L_{j}$ is (the image-set of) a singular 2-cell with no singularities on its boundary.

To see this, consider the above sets in the order $1,5,2,3,4,6$.

LEMMA 9.1.2. $L_{j} \cap \mathrm{Bd} S \subset$ Int $A_{4 j+2}^{\prime}$ for each $j$.

Proof of LEMMA. Bd $S \cap(1)=\varnothing$, because $\mathbf{B}^{\prime}$ is a barrier system for $S$. $\operatorname{Bd} S \cap(2)=\varnothing$, because (2) $\subset A_{1, j} \subset \operatorname{Bd} S_{1}$. Bd $S \cap(4)=\varnothing$, because (4) $\subset$ Bd $S_{2}$. Bd $S \cap(5)=\varnothing$, because Bd $S \cap \operatorname{Bd} C_{4 j+2}^{\prime} \subset d_{4 j+2}^{+} \cup d_{4 j+2}^{-}$. Bd $S \cap$ (6) $=\varnothing$, because Bd $S \cap\left(d_{4 j+2}^{\prime+} \cup d_{4 j+2}^{\prime-}\right)=\varnothing$.

LEMMA 9.1.3. $L_{j} \cap C_{4 k}=\varnothing$ for each $k$.

By construction: recall that $A_{4 j+2}^{\prime} \cap C_{4 k}=\varnothing$, and

$$
A_{1, j}, A_{2, j} \subset \bigcap_{r} f^{r}\left(\text { Int } C_{4 j+2}\right) \text {. }
$$


LEMMA 9.1.4. Every two different sets $C_{4 r}, C_{4 s}$ lie in different components of $S_{0}-\cup_{j=1}^{m} L_{j}$.

Proof of Lemma. Suppose not. Then a point of some $C_{4 r}$ can be joined to a point of a different set $C_{4 s}$ by a broken line $b$ in $S_{0}-U_{j} L_{j}$. Now $L_{j}$ is a singular 2-cell with boundary Bd $A_{4 j+2} \cap \mathrm{Bd} S_{0}$. Since $S_{0} \cup \cup_{j} L_{j}$ is tame, we may assume for the moment that this set is polyhedral relative to $K(M)$, and apply the Dehn Lemma of C. Papakyriakopoulos. (See, for example, [MGT, p. 199].) Thus every neighborhood of $L_{j}$ contains a polyhedral 2-cell $D_{j}$ such that

$$
\text { Bd } D_{j}=\operatorname{Bd} A_{4 j+2} \cap \operatorname{Bd} S_{0}, \quad \text { Int } D_{j} \subset \operatorname{Int} S_{0} .
$$

Since $L_{j} \cap C_{4 k}=\varnothing$, and $L_{j} \cap b=\varnothing$, it follows that $D_{j}$ can be chosen for each $j$ so that $D_{j} \cap C_{4 k}=\varnothing$ for each $k$ and $D_{j} \cap b=\varnothing$. But this is impossible: obviously $\cup_{j} D_{j}$ decomposes $S_{0}$ into 3-cells each of which contains exactly one set $C_{4 k}$. (An elementary proof of this lemma is possible but tedious.)

LemMa 9.1.5. Every two different sets $\mathrm{Bd} S \cap C_{4 r}, \mathrm{Bd} S \cap C_{4 s}$ lie in different components of $\left(S_{1}-\operatorname{Int} S_{2}\right)-\cup_{j} A_{4 j+2}^{\prime}$.

Proof of lemma. By Lemma 9.1.4, every connected set $W$ in $\operatorname{Bd} S$ that intersects two different sets $C_{4 r}, C_{4 s}$ must intersect $L_{j}$ for some $j$. By Lemma 9.1.2, $W$ intersects Int $A_{4 j+2}^{\prime}$. Therefore no component of $\mathrm{Bd} S-\cup_{j} A_{4 j+2}^{\prime}$ intersects two different sets $C_{4 r}, C_{4 s}$. Since there are exactly $m$ sets $C_{4 r}$, and exactly $m$ components of $\left(S_{1}-\right.$ Int $\left.S_{2}\right)-\cup_{j} A_{4 j+2}^{\prime}$, each of the former sets must lie in one of the latter; and the lemma follows.

Thus $S$ has the following properties.

(1) $S \subset$ Int $S_{1}, S_{2} \subset$ Int $S$.

(2) $S$ and B satisfy the conditions for $S_{1}$ and B in Theorem 8.1.

(Since $S$ has no cellular oscillations in $\mathbf{B}^{\prime}$, it follows that $S$ has no cellular oscillations in B. Since $S \subset$ Int $S_{1}, S$ is "sufficiently small" so that $S$ and B' satisfy the other conditions for $S_{1}$ and B in Theorem 8.1. Therefore so also do $S$ and B. But (2) as stated is what we need in Theorem 9.1. And the stronger condition using $\mathbf{B}^{\prime}$ would not necessarily be preserved by certain hypothetical operations presently to be discussed.)

(3) Different sets Bd $S \cap C_{4 k}^{\prime}$ lie in different components of $\left(S_{1}-\operatorname{Int} S_{2}\right)$ $-\cup_{j} A_{4 j+2}^{\prime}$.

Evidently we may assume also that

(4) $\mathrm{Bd} S$ is in general position relative to each set $A_{4 j+2}^{\prime}$.

(If not, we move $\operatorname{Pr}$ Int $A_{4 j+2}^{\prime}$ into general position relative to $\operatorname{Pr} \operatorname{Bd} S$, and lift.)

Finally we may suppose that $S$ is chosen, subject to all the above 
conditions, so as to minimize the total number of components of all sets Bd $S \cap$ Int $A_{4 j+2}^{\prime}$. On this basis, we shall show that $S$ is as in the conclusion of Theorem 9.1.

LeMMA 9.1.6. Let $J$ be a component of $\mathrm{Bd} S \cap$ Int $A_{4 j+2}^{\prime}$. If $J$ bounds a 2-cell in $\mathrm{Bd} S$, then $J$ bounds a 2-cell in $A_{4 j+2}^{\prime}$.

Proof of LEMMA. Suppose not. Then $J$ separates the two components of Bd $A_{4 j+2}^{\prime}$ from one another in $A_{4 j+2}^{\prime}$. It follows immediately that the latitudinal polygon $\mathrm{Bd} A_{4 j+2}^{\prime} \cap \mathrm{Bd} S_{1}$ is contractible in $S_{1}-F$, which is absurd.

LEMMA 9.1.7. Let $J$ be a component of $\mathrm{Bd} S \cap$ Int $A_{4 j+2}^{\prime}$. If $J$ bounds a 2-cell in $A_{4 j+2}^{\prime}$, then $J$ bounds a 2-cell in $\mathrm{Bd} S$.

Proof of LEMMA. Since Bd $S$ contains a latitudinal polygon disjoint from $J$ (Theorem 6.3), it follows that $J$ is latitudinal in $S$ or bounds a 2-cell in $\operatorname{Bd} S$. The former is impossible, because $J$ is contractible in $S-F$. The lemma follows.

Lemma 9.1.8. No component of $\mathrm{Bd} S \cap \operatorname{Int} A_{4 j+2}^{\prime}$ bounds a 2-cell in $A_{4 j+2}^{\prime}$.

Proof of Lemma. Suppose that $J=\operatorname{Bd} D_{J}, D_{J} \subset A_{4 j+2}^{\prime}$. Then $J$ bounds a 2-cell $D_{J}^{\prime} \subset \mathrm{Bd} S$, since otherwise a latitudinal polygon in $\operatorname{Bd} S$ would be contractible in $S_{1}-F$. Now $f$ is of period $n$, and both Bd $S$ and $A_{4 j+2}^{\prime}$ are $f$-invariant. We cannot have $f\left(D_{J}\right)=D_{J}$, since $D_{J}$ contains no fixed point of $f$. And no set $f^{r}\left(D_{J}\right)$ can lie in the interior of another, since then the number of components of $\mathrm{Bd} S \cap \operatorname{Int} A_{4 j+2}^{\prime}$ would be infinite. Therefore the 2-cells $f^{r}\left(D_{J}\right)$ are disjoint, and are permuted by $f$. Thus $\operatorname{Pr} J$ is a component of $\operatorname{Pr} \operatorname{Bd} S \cap \operatorname{Pr} A_{4 j+2}^{\prime}$, and bounds 2-cells $\operatorname{Pr} D_{J}$ and $\operatorname{Pr} D_{J}^{\prime}$ in $\operatorname{Pr} A_{4 j+2}^{\prime}$ and $\operatorname{Pr} \mathrm{Bd} S$ respectively. We may suppose, without loss of generality, that $J$ is inmost in $A_{4 j+2}^{\prime}$, in the sense that Int $D_{J}$ contains no component of $\mathrm{Bd} S \cap$ $A_{4 j+2}^{\prime}$. It follows that Int $\operatorname{Pr} D_{J}$ contains no point of $\operatorname{Pr} \operatorname{Bd} S$. We substitute $\operatorname{Pr} D_{J}$ for $\operatorname{Pr} D_{j}^{\prime}$ in $\operatorname{Pr} \operatorname{Bd} S$, force the resulting surface slightly off $\operatorname{Pr} A_{4 j+2}^{\prime}$ in the neighborhood of $\operatorname{Pr} D_{J}$, and then move the resulting surface slightly (if need be), so as to restore general position relative to $\operatorname{Pr} \mathrm{Bd} C_{4 j+2}^{\prime}$. This gives a torus $T$. Since $\operatorname{Pr} \operatorname{Bd} S$ and $\operatorname{Pr} D_{J}$ lie in $\operatorname{Pr}\left(\operatorname{Int} S_{1}-S_{2}\right), T$ can be chosen so as to lie in $\operatorname{Pr}\left(\operatorname{Int} S_{1}-S_{2}\right)$.

Let $\tilde{T}=\operatorname{Pr}^{-1} T$. Since $\operatorname{Pr} \mid \tilde{T}$ is an $n$-sheeted covering of a torus, $\tilde{T}$ is a torus. Since $T$ is polyhedral relative to $K(U)$, it follows that $\tilde{T}$ is polyhedral relative to $K(\tilde{U})$. Let $S^{\prime}$ be the component of $M-\tilde{T}$ that contains $F$. By Theorem 4.10, $S^{\prime}$ is a solid torus. Since

$$
\text { Bd } S^{\prime}=\tilde{T} \subset \operatorname{Int} S_{1}-S_{2},
$$

it follows that

(1) $S^{\prime} \subset$ Int $S_{1}, S_{2} \subset$ Int $S$. 
We shall show that the other conditions for $S$ are also preserved by the operation $S \rightarrow S^{\prime}$.

Since B is a barrier system for $S_{1}$, and $S^{\prime} \subset S_{1}$, we have $S^{\prime} \subset$ Int $S_{0}$, and $A_{4 k} \cap S^{\prime}=\varnothing$ for each $k$. Also $\mathrm{Bd} S^{\prime}$ is in general position relative to each set Bd $C_{4 k}^{\prime}$. Therefore B is a barrier system for $S^{\prime}$. Since $\mathrm{Bd} S^{\prime}=\tilde{T}=\operatorname{Pr}^{-1} T$, it follows that $\mathrm{Bd} S^{\prime}$ is $f$-invariant. Therefore so also is $S^{\prime}$. We recall that $A_{4 j+2}^{\prime} \cap C_{4 k}=\varnothing$ for each $j$ and $k$. It follows that $\mathrm{Bd} S^{\prime} \cap C_{4 k}$ is the union of some or all of the components of $\mathrm{Bd} S \cap C_{4 k}$. Since $S$ has no cellular oscillations in B, it follows that $S^{\prime}$ has the same property; the point is that if $J$ is a polygon in $\mathrm{Bd} S^{\prime} \cap C_{4 k}^{\prime}$, and $J$ bounds a 2-cell $D_{J}$ in $\mathrm{Bd} S^{\prime}$, then $J \subset \operatorname{Bd} S$, and $J$ cannot be latitudinal in $S$, so that $J$ bounds a 2-cell $D_{J}^{\prime}$ in Bd $S$. Since $S$ has no cellular oscillations in $\mathbf{B}$, we have $D_{J}^{\prime} \subset \cap_{r} f^{r}\left(C_{4 k}\right)$. Therefore $D_{J}^{\prime} \subset \mathrm{Bd} S^{\prime}, D_{J}^{\prime}=D_{J}$, and $D_{J} \subset \cup_{r} f^{r}\left(C_{4 k}\right)$. Since $S_{1}$ is "sufficiently small" in the sense of Theorem 8.1, and $S^{\prime} \subset S_{1}$, it follows that $S^{\prime}$ is "sufficiently small." Thus we have:

(2) $S^{\prime}$ and B satisfy all the conditions for $S_{1}$ and B in Theorem 8.1.

It is easy to check, geometrically, that:

(3) Different sets Bd $S^{\prime} \cap C_{4 k}^{\prime}$ lie in different components of $\left(S_{1}-\operatorname{Int} S_{2}\right)$ $-\cup_{j} A_{4 j+2}^{\prime}$.

To see this, regard the operation $\operatorname{Pr} \mathrm{Bd} S \rightarrow T$ as an operation Bd $S \rightarrow \tilde{T}$. Condition (2) is unaffected by (i) the deletion of the 2-cells $f^{r}\left(D_{J}\right)$, (ii) the addition of the 2-cells $f^{r}\left(D_{J}^{\prime}\right)$, (iii) the "forcing off" process, and (iv) the restoration of general position.

Obviously the operation $S \rightarrow S^{\prime}$ preserves (4); the effect is to delete certain components of Bd $S \cap A_{4 j+2}^{\prime}$.

But all this is impossible: it preserves (1)-(4), and reduces the total number of components of all sets $\mathrm{Bd} S \cap A_{4 j+2}^{\prime}$. The lemma follows.

LEMMA 9.1.9. Each set $\mathrm{Bd} S \cap A_{4 j+2}^{\prime}$ has at most one component.

Proof OF LEMMA. We now know that every component of this set is latitudinal in $S$, and separates the components of $\mathrm{Bd} A_{4 j+2}^{\prime}$ from one another in $A_{4 j+2}^{\prime}$. Since every two of these components bound an annulus in $A_{4 j+2}^{\prime}$, and $f$ is periodic, it follows that each of them is $f$-invariant. Suppose that there are more than one of them. Then their union decomposes $\mathrm{Bd} S$ into annuli with disjoint interiors, appearing in a certain cyclic order on $\mathrm{Bd} S$. If each of these annuli approaches Int $A_{4 j+2}^{\prime}$ locally from both sides, then it follows by an easy geometric argument that the number of these annuli is infinite, which is false. Therefore there is an annulus $A_{s}$ in $\mathrm{Bd} S$, with $\mathrm{Bd} A_{s} \subset A_{4 j+2}^{\prime}$, such that $A_{s}$ approaches Int $A_{4 j+2}^{\prime}$ locally from only one side. Now $\operatorname{Bd} A_{s}$ bounds an annulus $A_{s}^{\prime}$ in Int $A_{4 j+2}^{\prime}$; and if two such annuli $A_{s}^{\prime}$ intersect, then one contains the other in its interior. Therefore we may choose $A_{s}$ in such a way 
that Int $A_{s}^{\prime} \cap \mathrm{Bd} S=\varnothing$. Automatically we have

$$
A_{s}^{\prime} \cap\left(\mathrm{Bd} S_{1} \cup \mathrm{Bd} S_{2}\right)=\varnothing \text {. }
$$

We now simplify in much the same way as for 2-cells. Since each component of $\operatorname{Bd} A_{s}=\operatorname{Bd} A_{s}^{\prime}$ is $f$-invariant, it follows that $\operatorname{Pr} A_{s}$ and $\operatorname{Pr} A_{s}^{\prime}$ are annuli (with the same boundary). In $\operatorname{Pr} \operatorname{Bd} S$, we replace $\operatorname{Pr} A_{s}$ by $\operatorname{Pr} A_{s}^{\prime}$, force the resulting surface off $\operatorname{Pr} \operatorname{Int} A_{4 j+2}^{\prime}$, and move the "new parts" of the new surface slightly, so as to get them into general position relative to $\operatorname{Pr} \mathrm{Bd} C_{4 i+2}^{\prime}$. Then we lift. As in the proof of the preceding lemma, this gives a torus $T$ which is the boundary of a solid torus $S^{\prime}$, and $S^{\prime}$ satisfies all the conditions for $S$; the verifications are substantially the same as in the preceding proof. As before, all this is impossible, because it reduces the total number of components of the sets $\mathrm{Bd} S \cap A_{4 j+2}^{\prime}$.

Lemma 9.1.10. Each set $\mathrm{Bd} S \cap A_{4 j+2}^{\prime}$ is a polygon.

Proof of LemMa. By (4) and the preceding lemma, it will be sufficient to show that $\mathrm{Bd} S \cap A_{4 j+2}^{\prime} \neq \varnothing$. If this is false, then

$$
\operatorname{Bd} S \cap A_{4 j+2}^{\prime}=\operatorname{Bd} S \cap L_{j}=\varnothing \text {. }
$$

It follows, as in the proof of Lemma 9.1.4, that $\mathrm{Bd} A_{4 j+2} \cap \mathrm{Bd} S_{0}$ bounds a tame 2-cell in $S_{0}-S$, so that $F$ lies in a 3-cell in $S_{0}$, and $F$ is contractible in $S_{0}$, which is absurd.

Now the polygons Bd $S \cap \operatorname{Int} A_{4 j+2}$ decompose Bd $S$ into annuli $N_{j}$, with

$$
\operatorname{Bd} N_{j}=\left(A_{4 j-2}^{\prime} \cup A_{4 j+2}^{\prime}\right) \cap \operatorname{Bd} S \text {. }
$$

LEMMA 9.1.11. $N_{j}$ intersects $C_{4 k}$ only if $k=j$.

Proof of LemMa. We know that the sets $L_{j}$ separate every two different sets $C_{4 k}$ from one another in $S_{0}$. By Theorem 7.1, for each $j$ there is an $f$-invariant latitudinal polygon $J_{j}$ in $\mathrm{Bd} S \cap \operatorname{Int} C_{4 j}$. Let $V_{j}$ be the component of $\mathrm{Bd} S-\cup{ }_{r} A_{4 r+2}^{\prime}=\mathrm{Bd} S-\cup, L_{r}$ that contains $J_{j}$. Then $\bar{V}_{j}$ is a 2-manifold with boundary, and $\mathrm{Bd} \bar{V}_{j}$ consists of one or both of the polygons $\mathrm{Bd} S \cap L_{j-1}$ and $\mathrm{Bd} S \cap L_{j}$. If $\mathrm{Bd} \bar{V}_{j}$ is connected, then the component of $S_{0}-\cup, L_{r}$ that contains $C_{4 j}$ intersects Bd $S$ in the union of two connected sets $V_{j}, V_{j}^{\prime}$, and the closure of each of these is a 2-cell with handles. Since Bd $S$ is a torus, that is, a 2-cell with only one handle, it follows that at least one of the sets $\bar{V}_{j}, \bar{V}_{j}^{\prime}$ is a 2-cell. It follows that a latitudinal polygon in $\mathrm{Bd} S_{0}$ bounds a 2-cell in $S_{0}-F$, which is impossible. Therefore $N_{j}=\bar{V}_{j}$ for each $j$, and the lemma follows.

Now for each $j$ let $J_{j}$ be an $f$-invariant latitudinal polygon in $\operatorname{Bd} S \cap C_{4 j}$, as in the proof of the preceding lemma. Then $J_{j} \subset \operatorname{Int} N_{j}$ for each $j$, and $J_{j}$ separates the two components of Bd $N_{j}$ from one another in $N_{j}$. Thus

$$
N_{j}=N_{j}^{-} \cup N_{j}^{+} \text {, }
$$


where $N_{j}^{-}$and $N_{j}^{+}$are annuli, and

For each $\mathrm{j}$, let

$$
N_{j}^{+} \cap N_{j}^{-}=J_{j}=\operatorname{Bd} N_{j}^{-} \cap \operatorname{Bd} N_{j}^{+} .
$$

$$
M_{j}=N_{j}^{+} \cup N_{j+1}^{-}
$$

Then $S$ and the sets $M_{j}$ satisfy the conclusion of Theorem 9.1 .

The notations $\mathbf{B}^{\prime}=\left[\mathbf{P}, \mathbf{C}, \mathbf{C}^{\prime}, \mathbf{A}, S_{0}\right], \mathbf{C}=\left\{C_{4 j}\right\}, \mathbf{C}^{\prime}=\left\{C_{4 j}^{\prime}\right\}$, and so on, in Theorem 9.1, were really part of the apparatus of the proof. The conclusion of the theorem mentions only $\mathbf{B}$, and not $\mathbf{B}^{\prime}$. Moreover, given any barrier system $\mathbf{B}$ for $F$, there is a barrier system $\mathbf{B}^{\prime}$ for $F$ such that $\mathbf{B}$ and $\mathbf{B}^{\prime}$ satisfy the conditions of Theorem 9.1. The theorem can therefore be restated more simply, as follows.

Theorem 9.2. Let $\mathbf{B}=\left[\mathbf{P}, \mathbf{C}, \mathbf{C}^{\prime}, \mathbf{A}, S_{0}\right]$ be a barrier system for $F$, with $\mathbf{P}=\left\{P_{j}\right\}, \mathbf{C}=\left\{C_{j}\right\}, \mathbf{C}^{\prime}=\left\{C_{j}^{\prime}\right\}, \mathbf{A}=\left\{A_{j}\right\}(1 \leqslant j \leqslant m)$. Then every neighborhood of $F$ contains a solid torus $S$ such that

(1) $S$ and $\mathbf{B}$ satisfy all the conditions for $S_{1}$ and $\mathbf{B}$ in Theorem 8.1, and

(2) Bd $S$ is the union of a collection of annuli $M_{j}(1 \leqslant j \leqslant m)$, intersecting only on their boundaries, such that

(2.1) $M_{j}$ is f-invariant,

(2.2) $\mathrm{Bd} M_{j}$ is the union of a latitudinal polygon in $S \cap C_{j}$ and a latitudinal polygon in $S \cap C_{j+1}$, and

(2.3) $M_{j}$ intersects $C_{k}$ only if $k=j$ or $k=j+1$.

TheOREM 9.3. In Theorem $9.2, \delta M_{j} \leqslant 5 \delta \mathrm{B}$.

Because $M_{j}$ lies in the union of $C_{j}, C_{j+1}$, and the three components of $S_{0}-\cup_{r} C_{r}$ that are contiguous to these two sets.

TheOREM 9.4. Let $\mathbf{B}$ and $\mathbf{B}^{\prime}$ be barrier systems for $F$, and let $S$ be a solid torus such that $S$ and $\mathbf{B}^{\prime}$ satisfy all the conditions for $S$ and $\mathbf{B}$ in Theorem 9.2. If the mesh $\delta \mathbf{B}^{\prime}$ of $\mathbf{B}^{\prime}$ is sufficiently small, then $S$ has no cellular oscillations in $\mathbf{B}$.

Proof. Given $\mathbf{B}=\left[\mathbf{P}, \mathbf{C}, \mathbf{C}^{\prime}, \mathbf{A}, S_{0}\right]$, consider the pairs $C_{j} \in \mathbf{C}, C_{j}^{\prime} \in \mathbf{C}^{\prime}$. If $\delta \mathbf{B}^{\prime}$ is sufficiently small, then

$$
S \cap C_{j}^{\prime} \subset \bigcap_{r} f^{r}\left(\text { Int } C_{j}\right) .
$$

If $\delta \mathbf{B}^{\prime}$ is sufficiently small, then no annulus $M_{j} \subset \mathrm{Bd} S$ (as in Theorem 9.2) intersects both a set $C_{j}^{\prime}$ and the corresponding set $M-\cap_{r} f^{r}\left(\right.$ Int $\left.C_{j}\right)$.

If $\delta \mathbf{B}^{\prime}$ is small in both these senses, then every polygon $J$ in $\mathrm{Bd} S \cap C_{j}^{\prime}$ lies in an annulus $A$ in $\mathrm{Bd} S \cap \cap{ }_{r} f^{r}\left(\right.$ Int $C_{j}$ ), namely, the union $A$ of all the annuli $M_{j}$ that intersect $J$. If $J$ bounds a 2-cell in $S$, then $J$ bounds a 2-cell in $A$, and the theorem follows. 
10. Transverse solid tori. If $B$ and $\mathbf{B}^{\prime}$ are related as in Theorem 9.1, then we shall say that $\mathbf{B}^{\prime}$ is a 4-subdivision of $\mathbf{B}$.

THEOREM 10.1. There are sequences $\mathbf{B}_{1}, \mathbf{B}_{2}, \ldots ; \mathbf{B}_{1}^{\prime}, \mathbf{B}_{2}^{\prime}, \ldots ; S_{1}, S_{2}, \ldots$ such that the following conditons hold.

(1) For each $i, \mathbf{B}_{i}$ is a barrier system $\left[\mathbf{P}_{i}, \mathbf{C}_{i}, \mathbf{C}_{i}^{\prime}, \mathbf{A}_{i}, S_{0, i}\right]$ for $F$, and $\mathbf{B}_{i}^{\prime}$ is a 4-subdivision [ $\left.{ }^{\prime} \mathbf{P}_{i}, \mathbf{C}_{i},{ }^{\prime} \mathbf{C}_{i}^{\prime}, \mathbf{A}_{i}, S_{0, i}\right]$ of $\mathbf{B}_{i}$, so that $\mathbf{P}_{i}=\left\{\boldsymbol{P}_{i, 4 j}\right\}, \quad \mathbf{P}_{i}=\left\{\boldsymbol{P}_{i k}\right\}$ $\left(1<j<m_{i}, 1<k \leqslant 4 m_{i}\right)$, and similarly for $\mathbf{C}_{i}, \mathbf{C}_{i}^{\prime}$, and so on.

(2) For each $i, \mathbf{P}_{i} \subset \mathbf{P}_{i+1}$.

(3) $\operatorname{Lim}_{i \rightarrow \infty} \delta_{i}=\operatorname{Lim}_{i \rightarrow \infty} \delta \mathbf{B}_{i}=0$.

(4) For each $i, S_{i}$ and $\mathbf{B}_{i}^{\prime}$ satisfy the conditions for $S$ and $\mathbf{B}$ in Theorem 9.2.

(5) For each $i, S_{i+1} \subset$ Int $S_{i}$.

(6) For each $i$ there is a collection $\left\{A_{i j}^{\prime}\right\}\left(1 \leqslant j \leqslant m_{i}\right)$ of disjoint annuli, such that

(6.1) $A_{i j}^{\prime}$ is f-invariant,

(6.2) $A_{i j}^{\prime} \subset S_{i}-$ Int $S_{i+1}$,

(6.3) $A_{i j}^{\prime} \cap\left(\mathrm{Bd} S_{i} \cup \mathrm{Bd} S_{i+1}\right)=\operatorname{Bd} A_{i j}^{\prime}$, and this set is the union of a latitudinal polygon in $S_{i}$ and a latitudinal polygon in $S_{i+1}$,

(6.4) Bd $A_{i j}^{\prime} \subset$ Int $C_{4 j}^{\prime}$,

(6.5) $A_{i j}^{\prime}$ intersects $C_{i k}$ only if $k=4 j$ or $k=4 j \pm 1$, and

(6.6) $A_{i j}^{\prime}$ is polyhedral relative to $K(\tilde{U})$.

Proof. Obviously, by repeated applications of Theorem 6.1, we can get sequences $\mathbf{B}_{1}, \mathbf{B}_{2}, \ldots$ and $\mathbf{B}_{1}^{\prime}, \mathbf{B}_{2}^{\prime}, \ldots$ satisfying (1), (2), and (3); and we can make the diameters $\delta_{i}=\delta B_{i}$ as small as we please.

We define the three desired sequences recursively, as follows.

(i) Define $\mathbf{B}_{1}, \mathbf{B}_{1}^{\prime}$, with $\delta \mathbf{B}_{1}<1$. By Theorem 9.2 there is an $S_{1}$ such that $S_{1}$ and $B_{1}^{\prime}$ satisfy the conditions for $S$ and $B$ in Theorem 9.2. We take $S_{1}$ in a sufficiently small neighborhood of $F$ so that $S_{1}$ and $\mathbf{B}_{1}^{\prime}$ satisfy the conditions for $S_{1}$ and $\mathrm{B}$ in Theorem 8.1.

(ii) Suppose that we have given $\mathbf{B}_{i}, \mathbf{B}_{i}^{\prime}$, and $S_{i}$ for $i \leqslant i_{0}$, and $\left\{A_{i j}^{\prime}\right\}$ for $i<i_{0}$, satisfying (1)-(6). Define $\mathbf{B}_{i_{0}+1}, B^{\prime}{ }_{i_{0}+1}$ so as to preserve (1) and (2), making $\delta_{i_{0}+1}=\delta B_{i_{0}+1}$ sufficiently small so that if $S^{\prime}$ and $B_{i_{0}+1}^{\prime}$ satisfy the conditions for $S$ and B in Theorem 9.2, then $S^{\prime}$ has no cellular oscillations in $\mathbf{B}_{i_{0}}^{\prime}$. (By Theorem 9.4, this can be done.) By the induction hypothesis, $S_{i_{0}}$ and $\mathbf{B}_{i_{0}}^{\prime}$ satisfy the conditions for $S_{1}$ and B in Theorem 8.1. Let $S_{i_{0}+1}$ be a solid torus such that $S_{i_{0}+1}$ and $\mathbf{B}_{i_{0}+1}^{\prime}$ satisfy the conditions for $S$ and $\mathbf{B}$ in Theorem 9.2. Then $S_{i_{0}}, S_{i_{0}+1}$, and $\mathbf{B}_{i_{0}}^{\prime}$ satisfy the conditions for $S_{1}, S_{2}$, and $\mathbf{B}$ in Theorem 8.1. Applying Theorem 8.1, we get a collection $\left\{A_{j}^{\prime}\right\}$ of annuli. Of these, we use only the annuli $A_{4 j}^{\prime}=A_{i_{0} j}^{\prime}$. By (5) of Theorem 8.1, different sets $A_{i_{0 j} j}^{\prime}$ are disjoint. (6.1)-(6.6) now follow directly from (1)-(6) of Theorem 8.1. 
Hereafter, the sequences described in Theorem 10.1 will be regarded as fixed.

THEOREM 10.2. For each $i, j$,

$$
A_{i j}^{\prime} \subset N\left(P_{i, 4 j}, 3 \delta_{i}\right) .
$$

This is true because $A_{i j}^{\prime}$ lies in the union of $C_{i, 4 j}$ and the two components of $S_{0, i}-\cup_{r} C_{i, 4 r}$ that are contiguous to $C_{i, 4 j}$.

THEOREM 10.3. For each $i, \mathrm{Bd} S_{i}$ is the union of a collection of annuli $M_{i j}$ $\left(1<j<m_{i}\right)$, intersecting only on their boundaries, such that (1) $M_{i j}$ is $f$-invariant, (2) Bd $M_{i j}$ is the union of a latitudinal polygon in $S_{i} \cap \operatorname{Int} C_{i, 4 j}$ and a latitudinal polygon in $S_{i} \cap$ Int $C_{i, 4 j+4}$, and (3) $M_{i j}$ intersects $C_{i k}$ only for $4 j<k<4 j+4$. Moreover, Bd $S_{i}$ is the union of a collection $\left\{N_{i j}\right\}$ of annuli, intersecting only on their boundaries, such that (4) for each $i, j, \operatorname{Bd} S_{i} \cap C_{i, 4 j}$ $\subset$ Int $N_{i j}$ and (5) $N_{i j}$ intersects $C_{i k}$ only for $4 j-2<k<4 j+2$.

Proof. Let $\left\{M_{j}^{\prime}\right\}$ be a collection of annuli $\left(1 \leqslant j<4 m_{i}\right)$ such that $M_{j}^{\prime}, S_{i}$, and $\mathbf{B}_{i}^{\prime}$ satisfy the conditions of Theorem 9.2. Let

$$
M_{i j}=\bigcup_{i=4 j}^{4 j+3} M_{j}^{\prime}, \quad N_{i j}=\bigcup_{r=4 j-2}^{4 j+1} M_{j}^{\prime} .
$$

For each $i, j$, let $J_{i j}=M_{i, j-1} \cap M_{i j}$. For each $i, j$, there is exactly one $k=k(i, j)$ such that $P_{i+1, k}=P_{i, 4 j}$.

TheOREM 10.4. For each $i, j\left(1 \leqslant j \leqslant m_{i}\right), J_{i j} \cup J_{i+1, k}$ is the boundary of an annulus $A_{i j}^{\prime \prime}$ such that

(1) $A_{i j}^{\prime \prime}$ is a polyhedron relative to $K(\tilde{U})$,

(2) $A_{i j}^{\prime \prime}$ is f-invariant,

(3) Int $A_{i j}^{\prime \prime} \subset$ Int $S_{i}-S_{i+1}$,

(4) $A_{i j}^{\prime \prime}$ intersects $C_{i, 4 r}$ only if $r=j$, and

(5) $A_{i j}^{\prime \prime} \subset N\left(P_{i, 4 j}, 4 \delta_{i}\right)$.

Moreover, the annuli $A_{i j}^{\prime \prime}$ can be chosen so as to be disjoint.

Proof. We start with the annuli $A_{i j}^{\prime}$ of Theorem 10.1, so that

$$
\text { Bd } A_{i j}^{\prime} \subset \operatorname{Int} C_{i, 4 j}^{\prime} \text {. }
$$

It follows that

$$
J_{i j}^{\prime}=A_{i j}^{\prime} \cap \operatorname{Bd} S_{i} \subset \operatorname{Int} N_{i j}, \quad N_{i j} \cap A_{i k}^{\prime}=\varnothing \quad \text { for } k \neq j .
$$

By Theorem 10.3, we have $J_{i j} \subset \operatorname{Int} N_{i j}$. Therefore $\operatorname{Pr} J_{i j}$, Pr $J_{i j}^{\prime} \subset \operatorname{Pr} \operatorname{Int} N_{i j}$. Since $\operatorname{Pr} N_{i j}$ is an annulus, it follows by Theorem 4.11 that there is a PL homeomorphism $h_{i j}: U \leftrightarrow U, \operatorname{Pr} N_{i j} \leftrightarrow \operatorname{Pr} N_{i j}$, such that $h_{i j} \mid \operatorname{Pr} \operatorname{Bd} N_{i j}$ is the identity and $h_{i j}\left(\operatorname{Pr} J_{i j}^{\prime}\right)=\operatorname{Pr} J_{i j}$; and $h_{i j}$ can be chosen so as to differ from the identity only in an arbitrarily small neighborhood of $\operatorname{Pr} \operatorname{Int} N_{i j}$. Thus we may 
assume that $h_{i j} \mid \operatorname{Pr} S_{i+1}$ and $h_{i j} \mid \operatorname{Pr}\left(\operatorname{Bd} S_{i}-N_{i j}\right)$ are identity mappings. Let

$$
A=h_{i j}\left(A_{i, 4 j}^{\prime}\right), \quad \tilde{A}=\operatorname{Pr}^{-1} A \text {. }
$$

Let $W_{i j}$ be the set on which $h_{i j}$ differs from the identity. Since $N_{i j}$ lies in the union $V_{i j}$ of $C_{i, 4 j}$ and the two adjacent components of $S_{i}-U_{r} C_{i, 4 r}$, it follows that $W_{i j}$ can be chosen so that

$$
\tilde{W}_{i j}=\operatorname{Pr}^{-1} W_{i j} \subset V_{i j} \text {. }
$$

It follows that $\tilde{A}$ intersects $C_{i r}$ only if $4 j-2 \leqslant r \leqslant 4 j+2$.

Now consider the other component $A_{i j}^{\prime} \cap \operatorname{Bd} S_{i+1}$ of $\mathrm{Bd} A_{i j}^{\prime}$. This is also a component of $\mathrm{Bd} \tilde{A}$. Let $J_{i+1, k}^{\prime}=S_{i+1} \cap \mathrm{Bd} \tilde{A}$. Let $r_{0}$ and $r_{1}$ be the integers such that $P_{i, 4 j-2}=P_{i+1, r_{0}}$ and $P_{i, 4 j+2}=P_{i+1, r_{1}}$. Let

$$
N=\bigcup_{r=r_{0}}^{r_{1}} M_{i+1, r}
$$

Then $N$ is an annulus, and contains $C_{i, 4 j} \cap \mathrm{Bd} S_{i+1}$ in its interior. Thus

$$
J_{i+1, k}^{\prime}, J_{i k} \subset \text { Int } N, \quad N \cap \operatorname{Bd} A_{i k}^{\prime}=\varnothing \quad \text { for } k \neq j,
$$

and

$$
\operatorname{Pr} J_{i+1, k}^{\prime}, \quad \operatorname{Pr} J_{i k} \subset \operatorname{Pr} \text { Int } N .
$$

As before, we move $\operatorname{Pr} J_{i+1, k}^{\prime}$ onto $\operatorname{Pr} J_{i k}$ by a PL homeomorphism $h_{i j}^{\prime}$ : $U \leftrightarrow U, \operatorname{Pr}$ Int $N \leftrightarrow \operatorname{Pr} I n t N$, such that $h_{i j}^{\prime} \mid \operatorname{Pr}\left(\operatorname{Bd} S_{i+1}-N\right)$ is the identity and such that $h_{i j}^{\prime}$ differs from the identity only in a small neighborhood of Pr Int $N$. Since $N$ intersects $C_{i r}$ only if $r_{0} \leqslant r \leqslant r_{1}$, and $\tilde{A}$ has the same property, it follows that $h_{i j}^{\prime}$ can be chosen so that the set

$$
A_{i j}^{\prime \prime}=\operatorname{Pr}^{-1} h_{i j}^{\prime}(A)
$$

intersects $C_{i, 4 r}$ only if $r=j$. It follows that $A_{i j}^{\prime \prime}$ lies in the union $W$ of $C_{i, 4 j}$ and the components of $S_{i}-\cup_{r} C_{i, 4 r}$ that are contiguous to $C_{i, 4 j}$. Therefore $A_{i j}^{\prime \prime}$ lies in the closure of $N\left(P_{i, 4 j}, 3 \delta_{i}\right)$, and hence in $N\left(P_{i, 4 j}, 4 \delta_{i}\right)$. And if our homeomorphisms are chosen so that the sets on which they differ from the identity are disjoint, then for each $i$, different sets $A_{i j}^{\prime \prime}$ will be disjoint. This completes the proof of Theorem 10.4 .

Now the union of the annuli $A_{i j}^{\prime \prime}$ decomposes the toroidal shell $S_{i}-$ Int $S_{i+1}$ into $m_{i}$ 3-manifolds $S_{i j}$ with boundary, each of these being polyhedral relative to $K(\tilde{U})$. Each $S_{i j}$ is bounded by the union of (a) the annulus $M_{i j}$ in $\mathrm{Bd} S_{i}$, (b) an annulus in Bd $S_{i+1}$, and (c) the union of two successive annuli $A_{i j}^{\prime \prime}, A_{i, j+1}^{\prime \prime}$.

THEOREM 10.5. Each $S_{i j}$ is f-invariant.

Because Bd $S_{i j}$ is $f$-invariant.

ThEOREM 10.6. $S_{1}=\cup_{i j} S_{i j} \cup F$. 
ProOF. Evidently

$$
S_{1}=\bigcup_{i}\left[S_{i}-\operatorname{Int} S_{i+1}\right] \cup F .
$$

Since for each $i$ the sets $S_{i j}$ form a decomposition of $S_{i}-$ Int $S_{i+1}$, the theorem follows.

TheOREM 10.7. $\operatorname{Pr} S_{1}=\cup_{i, j} \operatorname{Pr} S_{i j} \cup \operatorname{Pr} F$.

Proof by Theorem 10.6.

THEOREM 10.8. For each $i, j, S_{i j} \subset N\left(P_{i, 4 j}, 5 \delta_{i}\right)$.

PRoof. Let $r_{0}$ and $r_{1}$ be the integers such that $P_{i, 4 j}=P_{i+1, r_{0}}, P_{i, 4 j+4}=$ $P_{i+1, r_{i}}$. Let

$$
M_{i j}^{\prime}=\bigcup_{r=r_{0}}^{r_{1}-1} M_{i+1, r}
$$

so that

$$
\mathrm{Bd} S_{i j}=M_{i j} \cup A_{i j}^{\prime \prime} \cup A_{i, j+1}^{\prime \prime} \cup M_{i j}^{\prime}
$$

Now $M_{i j}$ intersects $C_{i, 4 r}$ only for $r=j, j+1 ; A_{i j}^{\prime \prime}$ intersects $C_{i, 4 r}$ only for $r=j$; and $A_{i, j+1}^{\prime \prime}$ intersects $C_{i, 4 r}$ only for $r=j+1$. Applying Theorem 10.3 to the sets $M_{i+1, r} \subset M_{i j}^{\prime}$ and the collection $\mathbf{C}_{i+1}$, it is also easy to check that $M_{i+1, r}$ intersects $C_{i, 4 s}$ only for $s=j, j+1$. Thus $\mathrm{Bd} S_{i j}$ lies in the union $W$ of $C_{i, 4 j}, C_{i, 4 j+4}$, and the components of $S_{i}-\cup_{r} C_{i, 4 r}$ that are contiguous to $C_{i, 4 j}$ and $C_{i, 4 j+4}$. Now $\bar{W}$ is a 3-cell; and since Bd $S_{i j} \subset \bar{W}$, it follows that $S_{i j} \subset \bar{W}$. An easy computation gives $\bar{W} \subset N\left(P_{i, 4 j}, 5 \delta_{i}\right)$, and the theorem follows.

THEOREM 10.9. Each $S_{i j}$ is a solid torus.

Proof. We know (by the hypothesis of Theorem 1.1) that $F$ is the boundary of a tame 2-cell $D \subset \mathbf{S}^{3}$. Let $K\left(\mathbf{S}^{3}\right)$ be a triangulation of $\mathbf{S}^{3}$. Then there is a homeomorphism $h: \mathbf{S}^{3} \leftrightarrow \mathbf{S}^{3}$, mapping $D$ onto a set which is polyhedral relative to $K\left(\mathbf{S}^{3}\right)$; and obviously $h$ can be chosen so that $h(D) \subset$ $\tilde{U}$. (If not, shrink $h(D)$ onto a set of small diameter, and move the resulting set into $\tilde{U}$.) Let $K_{1}(\tilde{U})$ be a triangulation of $\tilde{U}$ in which each simplex is a linear subsimplex of a simplex of $K\left(\mathbf{S}^{3}\right)$. Then $D \cap \tilde{U}$ is semilocally tame in $\tilde{U}$ relative to $K_{1}(\tilde{U})$. By Theorem $3.4, D \cap \tilde{U}$ is tame in $\tilde{U}$ relative to $K_{1}(\tilde{U})$. By the Hauptvermutung, $D \cap \tilde{U}$ is tame in $\tilde{U}$ relative to $K(\tilde{U})$. By Theorem 3.4, there is a homeomorphism $g: \tilde{U} \leftrightarrow \tilde{U}$, such that $g(D \cap \tilde{U})$ is polyhedral relative to $K(\tilde{U})$; and $g$ may be chosen so that there is an extension $g^{\prime}$ : $\mathbf{S}^{3} \leftrightarrow \mathbf{S}^{3}$ of $g$, such that $g^{\prime} \mid F$ and $g^{\prime} \mid\left(\mathbf{S}^{3}-M\right)$ are identity mappings. Therefore we may assume that the $D$ that was given has the property that $D \cap \tilde{U}$ is polyhedral relative to $K(\tilde{U})$; and of course we may suppose that Int $D$ is in 
general position relative to $\mathrm{Bd} S_{i j}$. Subject to all these conditions, we choose $D$ so as to minimize the number $q$ of components of $D \cap \mathrm{Bd} S_{i j}$. Each of these components is of course a polygon.

LEMMA 10.9.1. $q>0$.

Proof of Lemma. If $q=0$, then $S_{i j}$ lies in a 3-cell in $\mathbf{S}^{3}-F$, and so $\mathrm{Bd} A_{i j}^{\prime \prime} \cap \mathrm{Bd} S_{i}$ is contractible in $\mathrm{S}^{3}-F$. Since this set is a latitudinal polygon in $S_{i}$, this contradicts Theorem 4.9.

LEMMA 10.9.2. No polygon $J$ in $D \cap \mathrm{Bd} S_{i j}$ bounds a 2-cell in $\mathrm{Bd} S_{i j}$.

Proof of lemma. Suppose that $J=\operatorname{Bd} D_{J}$, where $D_{J}$ is a 2-cell in $\operatorname{Bd} S_{i j}$; and suppose that $J$ is inmost in Bd $S_{i j}$, in the sense that Int $D_{J}$ contains no other component of $D \cap \operatorname{Bd} S_{i j}$. Then $D$ can be "forced off $\mathrm{Bd} S_{i j}$ in the neighborhood of $D_{J}$." This is impossible, because it reduces $q$.

Now let $J$ be a component of $D \cap \operatorname{Bd} S_{i j}$ which is inmost in $D$, so that $J$ bounds a 2-cell $D_{J} \subset$ Int $D$ such that Int $D_{J} \cap \mathrm{Bd} S_{i j}=\varnothing$. If Int $D_{J} \subset \mathrm{S}^{3}-$ $S_{i j}$, then $S_{i j}$ lies in a 3-cell in $\mathbf{S}^{3}-F$, which is impossible, as in the proof of Lemma 10.9.1. Therefore Int $D_{J} \subset$ Int $S_{i j}$. When $S_{i j}$ is split apart at $D_{J}$, the result is a 3-cell. Reidentifying, we find that $S_{i j}$ is either a solid torus (with $J$ a latitudinal polygon in $S_{i j}$ ) or a solid Klein Bottle. The latter is impossible, because $S_{i j}$ is a subspace of $S^{3}$; and Theorem 10.9 follows.

THEOREM 10.10. Each set $\operatorname{Pr} S_{i j}$ is a solid torus.

Proof. Evidently $\operatorname{Pr} S_{i j}$ is a 3-manifold with boundary, polyhedral relative to $K(U)$, and $\operatorname{Bd} \operatorname{Pr} S_{i j}=\operatorname{Pr} \operatorname{Bd} S_{i j}$ is a torus. Since $S_{i j}$ is a solid torus, there is a loop $L$ in Bd $\operatorname{Pr} S_{i j}$ which is contractible in $\operatorname{Pr} S_{i j}$ but not in Bd $\operatorname{Pr} S_{i j}$. By the Loop Theorem it follows that there is a PL 2-cell $\Delta_{i j}$ in $\operatorname{Pr} S_{i j}$ such that

$$
\operatorname{Bd} \Delta_{i j}=\Delta_{i j} \cap \operatorname{Bd} \operatorname{Pr} S_{i j} \text {, }
$$

and such that $\operatorname{Bd} \Delta_{i j}$ is not contractible in $\operatorname{Bd} \operatorname{Pr} S_{i j}$. Consider the set $\operatorname{Pr}^{-1} \Delta_{i j}$. Since Int $\Delta_{i j}$ and $\mathrm{Bd} \Delta_{i j}$ are locally Euclidean, and $\operatorname{Pr}$ is a local homeomorphism, it follows that (a) $\operatorname{Pr}^{-1} \mathrm{Bd} \Delta_{i j}$ is the union of $n$ disjoint polygons and (b) $\operatorname{Pr}^{-1}$ Int $\Delta_{i j}$ is the union of $n$ disjoint sets homeomorphic to Int $\Delta_{i j}$. Thus (c) $\operatorname{Pr}^{-1} \Delta_{i j}=\cup_{r=1}^{n} d_{r}$, where the sets $d_{r}$ are disjoint 2-cells, with

$$
d_{r} \cap \operatorname{Bd} S_{i j}=\operatorname{Bd} d_{r}, \quad \text { Int } d_{r} \subset \operatorname{Int} S_{i j},
$$

so that the polygons Bd $d_{r}$ are latitudinal in $S_{i j}$. Thus the sets $d_{r}$ decompose $S_{i j}$ into $n$ 3-cells, each of which is mapped onto $\operatorname{Pr} S_{i j}$ under a mapping which identifies two disjoint 2-cells in its boundary. It follows, as in the proof of the preceding theorem, that $\operatorname{Pr} S_{i j}$ is either a solid torus or a solid Klein Bottle. Thus, to complete the proof of Theorem 10.10, it will be sufficient to show that $\operatorname{Pr} S_{i j}$ is orientable. 
Now Bd $S_{i j} \cap \operatorname{Bd} S_{i+1}$ is tame relative to $K(\tilde{U})$, and hence tame relative to a triangulation $K\left(\mathbf{S}^{3}\right)$ of $\mathbf{S}^{3}$; and every polygon $J$ in the annulus $A=S_{i j} \cap$ Bd $S_{i+1}$ either bounds a 2-cell in $A$ or is latitudinal in $S_{i+1}$. It follows, by a straightforward construction, that there is a tame 3-cell $C^{3}$ in $\mathbf{S}^{3}$ such that $C^{3} \cap C_{i j}=A$, and such that $\mathrm{Bd} C^{3} \cup \operatorname{Bd} S_{i j}$ is also tame. Thus the set $C_{1}^{3}=S_{i j} \cup C^{3}$ is a 3-cell. Let $g: C_{1}^{3} \leftrightarrow C_{1}^{3}$ be an extension of $f \mid S_{i j}$, such that $g$ is tame, and such that $g \mid C^{3}$ is conjugate to a rotation of a cylinder $\mathbf{B}^{2} \times[0,1]$ about its axis of symmetry. Thus the fixed-point set of $g$ is a broken line $B$, with its two end-points in the two components of $\mathrm{Bd} C_{1}^{3}-A$; and $B$ is unknotted in $C_{1}^{3}$, in the obvious sense. Now let $C_{2}^{3}$ be a combinatorial copy of $C_{1}^{3}$, with identifications such that $\mathrm{Bd} C_{2}^{3}=\mathrm{Bd} C_{1}^{3}$ and $C_{1}^{3} \cup C_{2}^{3}$ is a 3-sphere $S^{3}$. Now extend $g$ so as to get a homeomorphism $g^{\prime}: S^{3} \leftrightarrow S^{3}$, simplicial relative to some triangulation of $S^{3}$, and with an unknotted polygon as its fixed-point set. Any such $g^{\prime}$ is periodic, with the same period as $f$. The main result of $[\mathbf{M}]$ asserts that under these conditions $g^{\prime}$ is conjugate to a rotation. It follows that the orbit space $\Omega^{\prime \prime}$ of $g^{\prime}$ is a 3-sphere. Since $\operatorname{Pr} S_{i j}$ is homeomorphic to a subspace of $\Omega^{\prime \prime}$, it follows that $\operatorname{Pr} S_{i j}$ is orientable; and this is sufficient to complete the proof of Theorem 10.10.

THEOREM 10.11. In each set $\operatorname{Pr} S_{i j}$ there is a PL 2-cell $\Delta_{i j}$ such that

(1) Bd $\Delta_{i j}=\Delta_{i j} \cap \operatorname{Bd} \operatorname{Pr} S_{i j}$,

(2) Bd $\Delta_{i j}$ is latitudinal in $\operatorname{Pr} S_{i j}$,

(3) $\mathrm{Bd} \Delta_{i j}$ is in general position relative to each set $\operatorname{Pr} J_{r s}$ that lies in $\operatorname{Bd} \operatorname{Pr} S_{i j}$, and

(4) $\operatorname{Pr}^{-1} \Delta_{i j}$ is the union of $n$ disjoint 2-cells $d_{r}$, with $\mathrm{Bd} d_{r}=d_{r} \cap \operatorname{Bd} S_{i j}$.

Proof. In the proof of Theorem 10.10 we got 2-cells $\Delta_{i j}$ satisfying (1) and (4), such that Bd $\Delta_{i j}$ is not contractible in Bd $\operatorname{Pr} S_{i j}$. Therefore each $\Delta_{i j}$ also satisfies (2). These properties are preserved by any PL homeomorphism $\operatorname{Pr} S_{i j} \leftrightarrow \operatorname{Pr} S_{i j}$. By such a homeomorphism, we move each set Bd $\Delta_{i j}$ slightly so as to get condition (3).

In the following definition, the sets $A_{i j}^{\prime \prime}$ are as in Theorem 10.4.

Given $A_{i j}^{\prime \prime}$, let $j_{0}=j$; and for each $k \geqslant 0$ let $j_{k}$ be the integer such that $P_{i, 4 j_{0}}=P_{i+k, 4 j_{k}}$. (There is such a $j_{k}$; see (2) of Theorem 10.1.) We form the sequence

$$
A_{i, j_{0}}^{\prime \prime}, A_{i+1, j_{1}}^{\prime \prime}, \ldots
$$

and let

$$
D_{i j}=\bigcup_{k=0}^{\infty} A_{i+k j_{k}}^{\prime \prime} \cup\left\{P_{i, 4 j_{0}}\right\} .
$$

Under our conditions for the sets $A_{i j}^{\prime \prime}$, we have

$$
A_{i j_{k}}^{\prime \prime} \cap A_{i j_{k+1}}^{\prime \prime}=J_{i j_{k+1}} .
$$


By Theorem 10.7 we have

$$
A_{i+k j_{k}}^{\prime \prime} \subset N\left(P_{i+k, 4 j_{k}}, 4 \delta_{i+k}\right)=N\left(P_{i, 4 j_{0}}, 4 \delta_{i+k}\right) .
$$

Therefore

$$
\bigcup_{k=r}^{\infty} A_{i+k j_{k}}^{\prime \prime} \subset N\left(P_{i, 4 j_{0}}, 4 \delta_{i+r}\right)
$$

for each $r$. Therefore

$$
\mathrm{Cl}\left[\bigcup_{k=0}^{\infty} A_{i+k_{j} j_{k}}^{\prime \prime}\right]=\bigcup_{k=0}^{\infty} A_{i+k j_{k}}^{\prime \prime} \cup\left\{P_{i, 4 j_{0}}\right\} ;
$$

and it follows by a straightforward construction that $D_{i j}$ is a 2-cell. We recall that

$$
J_{i j}=M_{i, j-1} \cap M_{i j}=\operatorname{Bd} A_{i j}^{\prime \prime} \cap \operatorname{Bd} S_{i}
$$

Thus

$$
\operatorname{Bd} D_{i j}=J_{i j}
$$

for each $i, j$.

THEOREM 10.12. For each $i, j, J_{i j}$ is longitudinal in $S_{i j}$.

Proof. We have $J_{i j}=\operatorname{Bd} D_{i j}, D_{i j} \cap S_{i j}=A_{i j}^{\prime \prime} \subset \mathrm{Bd} S_{i j}$. It follows immediately that $J_{i j}$ is contractible in $M-\operatorname{Int} S_{i j}$. Now apply Theorem 4.9.

THEOREM 10.13. The 2-cells $\Delta_{i j}$ of Theorem 10.11 can be chosen in such a way that each intersection $\mathrm{Bd} \Delta_{i j} \cap \operatorname{Pr} J_{i j}$ is a single point.

Proof. Let $\left\{d_{r}\right\}$ be as in Theorem 10.11. Suppose that the sets $\Delta_{i j}$ are chosen (subject to the conditions of Theorem 10.11) so as to minimize the number of points in $\operatorname{Bd} \Delta_{i j} \cap \operatorname{Pr} J_{i j}$. On this basis we shall show that the theorem follows.

Now Bd $d_{r}$ is latitudinal in $S_{i j}, J_{i j}$ is longitudinal in $S_{i j}$, and these polygons are in general position relative to one another in Bd $S_{i j}$. By Theorem 4.6 it follows that these polygons cross each other algebraically once.

Suppose now that the theorem is false, so that $\operatorname{Bd} \Delta_{i j} \cap \operatorname{Pr} J_{i j}$ contains more than one point. It follows that $\mathrm{Bd} d_{r}$ intersects $J_{i j}$ in more than one point. From this we can easily show that there is a broken line $b_{r} \subset \operatorname{Bd} d_{r}$, and a broken line $b_{i j} \subset J_{i j}$, such that $b_{r} \cup b_{i j}$ bounds a 2-cell $d$ in Bd $S_{i j}$, with Bd $d \cap J_{i j}=b_{i j}$. Since different sets $d_{r}$ are disjoint, $J_{i j}$ is $f$-invariant, and $f$ is periodic, it follows that the images $f^{r}(d)$ are disjoint. Therefore $\operatorname{Pr} \mid d$ is a homeomorphism. Therefore $\operatorname{Pr} b_{r}$ can be dragged across $\operatorname{Pr} J_{i j}$, in the neighborhood of $\operatorname{Pr} d$, by a PL homeomorphism $\operatorname{Pr} S_{i j} \leftrightarrow \operatorname{Pr} S_{i j}, \operatorname{Bd} \operatorname{Pr} S_{i j} \leftrightarrow$ Bd Pr $S_{i j}$ which differs from the identity only in a small neighborhood of $d$. All this is impossible, because it reduces the number of components of $\operatorname{Bd} \Delta_{i j} \cap \operatorname{Pr} J_{i j}$. 
TheOREM 10.14. For each $i, j, \operatorname{Pr} J_{i j}$ is longitudinal in $\operatorname{Pr} S_{i j}$.

Proof. We know that Bd $\Delta_{i j}$ is latitudinal in $S_{i j}$, and that $J_{i j}$ crosses Bd $\Delta_{i j}$ in exactly one point. When we split $\operatorname{Pr} S_{i j}$ apart at $\Delta_{i j}$, $\operatorname{Pr} J_{i j}$ appears as a broken line joining points of the two 2-cells which have the same image when we reidentify. It follows that $\operatorname{Pr} J_{i j}$ carries a generator of $H_{1}\left(\operatorname{Pr} S_{i j}\right)$.

Theorem 10.15. Every polygon $\operatorname{Pr} J_{r s}$ that lies in $\operatorname{Bd} \operatorname{Pr} S_{i j}$ is longitudinal in $\operatorname{Pr} S_{i j}$.

Proof. There are finitely many such polygons; they are disjoint; none are contractible in Bd $\operatorname{Pr} S_{i j}$; and one of them $\left(\operatorname{Pr} J_{i j}\right)$ is longitudinal. Therefore so also are the others. (Theorem 4.3.)

11. A triangulation of $\Omega$. For each $i$, we define a sequence $S_{i, j, 0}, S_{i, j, 1}, \ldots$, inductively, as follows. (1) Let $S_{i, j, 0}=S_{i j}$. (2) Given a solid torus $S_{i j k} \subset S_{i+k}$ - Int $S_{i+k+1}$, intersecting Bd $S_{i+k+1}$ in an annulus which forms a union of annuli $M_{i+k+1, r}$, let $S_{i, j, k+1}$ be the union of all solid tori $S_{i+k+1, r}$ which have an annulus in common with $S_{i j k}$.

For each $i$, $j$, let $B_{i j}$ be the "short arc" in $F$ between $P_{i, 4 j}$ and $P_{i, 4 j+4}$ that is, the arc which contains no third point $P_{i, 4 r}$. Let

$$
D_{i j}=\bigcup_{k=0}^{\infty} S_{i j k} \cup B_{i j} .
$$

THEOREM 11.1. Each set $D_{i j}$ is compact.

Proof. By induction on $i$, it is easy to check that if $S_{i+k, r} \subset S_{i j k}$, then $P_{i+k, 4 r} \in B_{i j}$. By Theorem 10.8 it follows that

$$
S_{i+k, r} \subset N\left(P_{i+k, 4 r}, 5 \delta_{i+k}\right) \subset N\left(B_{i j}, 5 \delta_{i+k}\right),
$$

where $N(X, \varepsilon)$ is the $\varepsilon$-neighborhood of the set $X$. Since the sequence $\delta_{1}, \delta_{2}, \ldots$ is decreasing, it follows that

(a) $\cup_{k=k_{0}}^{\infty} S_{i j k} \subset N\left(B_{i j}, 5 \delta_{i+k}\right)$ for each $k_{0}$. Since $\cup_{k=0}^{k_{0}} S_{i j k}$ is closed for each $k_{0}$, it follows that

$$
\mathrm{Cl}\left[\bigcup_{k=0}^{\infty} S_{i j k}\right]=\bigcup_{k=0}^{\infty} S_{i j k} \cup B_{i j},
$$

and the theorem follows.

THEOREM 11.2. For each $i, j, \delta B_{i j} \leqslant 3 \delta_{i}$.

(Because $B_{i j}$ lies in the union of $C_{i, 4 j}, C_{i, 4 j+4}$, and the component of $S_{0, i}-\cup_{r} C_{i, 4 r}$ that lies between them, and each of these sets has diameter no greater than $\delta_{i}$.) 
THEOREM 11.3. $\operatorname{Lim}_{i \rightarrow \infty} \delta D_{i j}=0$, uniformly with respect to $j$. That is, for every $\varepsilon>0$ there is an $i_{0}$ such that if $i \geqslant i_{0}$, then $\delta D_{i j}<\varepsilon$ for every $j$.

Proof. Setting $k=0$ in (a) above, we get $D_{i j} \subset N\left(B_{i j}, 5 \delta_{i}\right)$. If $i$ is sufficiently large, then $\delta B_{i j}<\varepsilon / 3,5 \delta_{i}<\varepsilon / 3$. It follows that $\delta D_{i j}<\varepsilon$, as desired.

For each $i, j$, let $D_{i j}^{\prime}=\operatorname{Pr} D_{i j}$.

THEOREM 11.4. $\operatorname{Lim}_{i \rightarrow \infty} \delta D_{i j}^{\prime}=0$, uniformly with respect to $j$.

This is true because $\operatorname{Pr}$ is uniformly continuous.

THEOREM 11.5. $\operatorname{Pr} S_{1}$ is a solid torus.

Proof. Let $F^{\prime}$ be the circle in the $x z$-plane in $\mathbf{R}^{3}$, with center at $(0,0,0)$ and radius 2 . For each $i$, let $D_{i}$ be the closed circular region in the $x y$-plane, with center at $(2,0,0)$ and radius $1 / i$; and let $S_{i}^{\prime}$ be the solid of revolution obtained by rotating $D_{i}$ about the $y$-axis. Thus $S_{i}^{\prime}$ is a solid torus with $F^{\prime}$ as a spine, and $\cap_{i} S_{i}^{\prime}=F^{\prime}$.

Let $\mathbf{P}_{1}^{\prime}$ be a set of $m_{1}$ points $P_{1,4 j}^{\prime}$ of $F^{\prime}$, equally spaced on $F^{\prime}$. (We recall that $m_{i}$ is the number of points in $\mathbf{P}_{i}$.) Given an ascending sequence $\mathbf{P}_{1}^{\prime}, \mathbf{P}_{2}^{\prime}, \ldots, \mathbf{P}_{i}^{\prime}$, such that $\bigcup_{k=1}^{i} \mathbf{P}_{k}^{\prime}$ is the image of $\cup_{k=1}^{i} \mathbf{P}_{k}$ under a bijection $\mathbf{P}_{k} \leftrightarrow \mathbf{P}_{k}^{\prime}$ which preserves cyclic order on $F$ and $F^{\prime}$, we form $\mathbf{P}_{i+1}^{\prime}$ so as to preserve this property, in such a way that the points of $\mathbf{P}_{i+1}^{\prime}$ that lie on each "short arc" from $P_{i, 4 j}^{\prime}$ to $P_{i, 4 j+4}^{\prime}$ are equally spaced on the short arc. Thus the bijection $\mathbf{P}_{i} \leftrightarrow \mathbf{P}_{i}^{\prime}$ can be extended to give a bijection $\mathbf{P}_{i+1} \leftrightarrow \mathbf{P}_{i+1}^{\prime}$, preserving cyclic order. By (2) of Theorem 10.1, $\mathbf{P}_{i+1}^{\prime}$ decomposes $F^{\prime}$ into arcs which are at most one-fourth as long as the longest short arc of the type $P_{i, 4 j} P_{i, 4 j+4}$. It follows that $\cup_{i} \mathbf{P}_{i}^{\prime}$ is dense in $F^{\prime}$.

For each $i, j$, let $H_{i j}$ be the half-plane with the $y$-axis as edge, passing through $P_{i, 4 j}^{\prime}$. Then for each $i,\left\{H_{i j}\right\}$ decomposes Bd $S_{i}^{\prime}$ into annuli $M_{i j}^{\prime} ; H_{i j}$ intersects $S_{i}^{\prime}-$ Int $S_{i+1}^{\prime}$ in an annulus $A_{i j}^{\prime \prime \prime}$; and the sets $A_{i j}^{\prime \prime \prime}$ decompose $S_{i}^{\prime}-$ Int $S_{i+1}^{\prime}$ into solid tori $S_{i j}^{\prime}$.

It is a straightforward matter to define a triangulation $K\left(S_{1}^{\prime}\right)$ of $S_{1}^{\prime}$ (obviously not rectilinear in $\mathrm{R}^{3}$ ) such that (1) $F^{\prime}$ forms a subcomplex of $K\left(S_{1}^{\prime}\right)$ and (2) each set $M_{1, j}^{\prime}$ forms a subcomplex of $K\left(S_{1}^{\prime}\right)$. Evidently $K\left(S_{1}^{\prime}\right)$ gives a PL structure in $S_{1}^{\prime}$.

Evidently there is a homeomorphism

$$
h_{1}: \operatorname{Bd} S_{1}^{\prime} \leftrightarrow \operatorname{Bd} \operatorname{Pr} S_{1},
$$

such that

$$
h_{1}\left(M_{1, j}^{\prime}\right)=\operatorname{Pr} M_{1, j} \quad\left(1 \leqslant j \leqslant m_{1}\right) \text {; }
$$

and $h_{1}$ can be constructed so as to be PL relative to $K\left(S_{1}^{\prime}\right)$ and $K(U)$. 
Construction. For each $i, j$, let

$$
J_{i j}^{\prime}=M_{i, j-1}^{\prime} \cap M_{i j}^{\prime}=\operatorname{Bd} A_{i j}^{\prime \prime \prime} \cap \operatorname{Bd} S_{i}^{\prime} .
$$

First define $h_{1}$ on $\cup_{j} J_{1, j}^{\prime}$, so that $h_{1}\left(J_{1, j}^{\prime}\right)=\operatorname{Pr} J_{1, j}$ for each $j$. Then, in each set $M_{1, j}^{\prime}$, extend $h_{1}$ to two disjoint broken lines in $M_{1, j}^{\prime}$, joining the two components of $\mathrm{Bd} M_{1, j}^{\prime}$, and intersecting $\mathrm{Bd} M_{1, j}^{\prime}$ only in their end-points; these are mapped by the extended $h_{1}$ onto broken lines in the corresponding sets $\operatorname{Pr} M_{1, j}$. It is easy to ensure that the extended $h_{1}$ is PL. Now each of the sets $M_{1, j}^{\prime}, \operatorname{Pr} M_{1, j}$ is decomposed into two combinatorial 2-cells; and for appropriate choice of the initial $h_{1}$ ("preserving orientation"), the boundaries of the two 2-cells in $M_{1, j}^{\prime}$ will be mapped onto the boundaries of the two 2-cells in $\operatorname{Pr} M_{1, j}$. Therefore $h_{1}$ can be extended again so as to give the desired PL homeomorphism $h_{1}: \operatorname{Bd} S_{1}^{\prime} \leftrightarrow \operatorname{Bd} \operatorname{Pr} S_{1}$.

In further extensions of $h_{1}$, we shall not care whether our mappings are PL.

The $x z$-plane intersects each set $S_{i j}^{\prime}$ in the union of two 2-cells $d_{i, j, 1}^{\prime}, d_{i, j, 2}^{\prime}$, with $d_{i, j, 1}^{\prime}$ lying inside $F^{\prime}$ and $d_{i, j, 2}^{\prime}$ lying outside $F^{\prime}$. (The geometry here is clear, and so we need not list the ways in which these 2-cells intersect the sets already defined in $S_{1}^{\prime}$.) We have already defined

$$
h_{1}: \operatorname{Bd} S_{1}^{\prime} \leftrightarrow \operatorname{Bd} \operatorname{Pr} S_{1} .
$$

Suppose that we have an extension

$$
h_{i}: S_{1}^{\prime}-\operatorname{Int} S_{i}^{\prime} \leftrightarrow \operatorname{Pr} S_{1}-\operatorname{Int} \operatorname{Pr} S_{i},
$$

such that

$$
h_{i}\left(J_{i j}^{\prime}\right)=\operatorname{Pr} J_{i j} \quad\left(1 \leqslant j \leqslant m_{i}\right)
$$

We then extend $h_{i}$ to all arcs

$$
b_{i j k}^{\prime}=d_{i j k}^{\prime} \cap d_{i, j+1, k}^{\prime} \subset A_{i, j+1}^{\prime \prime \prime},
$$

so that these are mapped onto disjoint broken lines $b_{i j k}$ in $\operatorname{Pr} A_{i j}^{\prime \prime}$, intersecting Bd $S_{i+1}$ in points $p_{i j k}$. For $k=1,2$, let $e_{i j k}$ be disjoint broken lines in the annulus $\operatorname{Bd} \operatorname{Pr} S_{i j} \cap \operatorname{Bd} \operatorname{Pr} S_{i+1}$, joining $p_{i j k}$ to $p_{i, j+1, k}$, and intersecting the boundary of the annulus only in their end-points. We define an extension $h_{i+1}$ of $h_{i}$ such that

$$
h_{i+1}\left(b_{i j k}^{\prime}\right)=b_{i j k}, h_{i+1}\left(d_{i j k}^{\prime} \cap \operatorname{Bd} S_{i+1}^{\prime}\right)=e_{i j k} .
$$

We choose the broken lines $e_{i j k}$ in such a way that (1) $h_{i+1}\left(\mathrm{Bd} d_{i j k}^{\prime}\right)$ is latitudinal in $\operatorname{Pr} S_{i j}$ and (2) each of the polygons $\cup_{j} e_{i j k}(k=1,2)$ intersects each set $\operatorname{Pr} J_{i+1, r}$ in exactly one point. For $k=1,2$ let $d_{i j k}$ be a polyhedral 2-cell in $\operatorname{Pr} S_{i j}$ such that

$$
\mathrm{Bd} d_{i j k}=h_{i+1}\left(\mathrm{Bd} d_{i j k}^{\prime}\right)
$$

we choose these 2-cells so that they are disjoint.

Now any homeomorphism between the boundaries of two cells can be 
extended so as to give a homeomorphism between the cells. Thus we can extend $h_{i+1}$ so that $h_{i+1}\left(A_{i j}^{\prime \prime \prime}\right)=A_{i j}^{\prime \prime}$ for each $j$. The arcs $d_{i j k}^{\prime} \cap \operatorname{Bd} S_{i+1}^{\prime}$ decompose each set Bd $S_{i+1}^{\prime} \cap \mathrm{Bd} S_{i j}^{\prime}$ into two 2-cells, each of which intersects each set $J_{i+1, r}^{\prime}$ in an arc. We extend $h_{i+1}$ to each of these 2-cells, in such a way that

$$
h_{i+1}\left(\operatorname{Bd} S_{i+1}^{\prime} \cap \operatorname{Bd} S_{i j}^{\prime}\right)=\operatorname{Bd} \operatorname{Pr} S_{i+1} \cap \operatorname{Bd} \operatorname{Pr} S_{i j} ;
$$

and we choose these extensions in such a way that $h_{i+1}\left(J_{i+1, r}^{\prime}\right)=\operatorname{Pr} J_{i+1, r}$ for each $r$. Now $d_{i, j, 1}^{\prime} \cup d_{i, j, 2}^{\prime}$ decomposes $S_{i j}^{\prime}$ into two 3-cells, and $h_{i+1}$ has already mapped the boundary of each of these onto a polyhedral 2-sphere in $\operatorname{Bd} \operatorname{Pr} S_{i j}$ $\cup d_{i, j, 1} \cup d_{i, j, 2}$. Thus $h_{i+1}$ can be extended to give

$$
\begin{aligned}
h_{i+1}\left(S_{i j}^{\prime}\right) & =\operatorname{Pr} S_{i j}, \\
h_{i+1}\left(S_{1}^{\prime}-\operatorname{Int} S_{i+1}^{\prime}\right) & =\operatorname{Pr} S_{1}-\operatorname{Int} \operatorname{Pr} S_{i+1}, \\
h_{i+1}\left(J_{i+1, r}^{\prime}\right) & =\operatorname{Pr} J_{i+1, r}
\end{aligned}
$$

for each $r$. Inductively, we get an ascending sequence $h_{1}, h_{2}, \ldots$ of homeomorphisms whose union is a homeomorphism

$$
h: S_{1}^{\prime}-F^{\prime} \leftrightarrow \operatorname{Pr} S_{1}-\operatorname{Pr} F .
$$

By continuity, we can extend $h$ to $F^{\prime}$, so that $h\left(S_{1}^{\prime}\right)=\operatorname{Pr} S_{1}$; the verification depends on Theorem 11.4.

Evidently the above proof proves more than the theorem, namely:

TheOREM 11.6. $\operatorname{Pr} S_{1}$ has a triangulation $K\left(\operatorname{Pr} S_{1}\right)$ such that (1) $\operatorname{Pr} F$ forms a subcomplex of $K\left(\operatorname{Pr} S_{1}\right)$ and (2) the identity mapping $\operatorname{Bd} \operatorname{Pr} S_{1} \leftrightarrow \operatorname{Bd} \operatorname{Pr} S_{1}$ is $\mathrm{PL}$ relative to $K\left(\operatorname{Pr} S_{1}\right)$ and $K(U)$.

Proof. Let $K\left(\operatorname{Pr} S_{1}\right)=\left\{\sigma \mid \sigma=h(\tau), \tau \in K\left(S_{1}^{\prime}\right)\right\}$.

TheOREM 11.7. $\Omega$ has a triangulation $K(\Omega)$ in which $\operatorname{Pr} F$ forms a subcomplex.

Proof. We have triangulations $K_{1}=K\left(\operatorname{Pr} S_{1}\right)$ and

$$
K_{2}=K\left(\Omega-\operatorname{Int} \operatorname{Pr} S_{1}\right)=\left\{\sigma \mid \sigma \in K(U), \sigma \subset \Omega-\operatorname{Int} \operatorname{Pr} S_{1}\right\} ;
$$

and the relation between these in $\operatorname{PL}$ in $\operatorname{Bd} \operatorname{Pr} S_{1}$. Thus the two induced triangulations of $\operatorname{Bd} \operatorname{Pr} S_{1}$ have a common subdivision; and this subdivision can be extended to give subdivisions $K_{1}^{\prime}, K_{2}^{\prime}$ of $K_{1}$ and $K_{2}$. Let $K(\Omega)=K_{1}^{\prime} \cup$ $K_{2}^{\prime}$.

THEOREM 11.8. $\Omega$ is a 3-manifold, and $\operatorname{Pr} F$ is tame in $\Omega$.

Proof by Theorems 11.5 and 11.7. 
12. Proof of Theorem 1.4. Let $M, K(M)$, and $F$ be as in Theorem 1.4, and let $D$ be a tame 2-cell such that $\mathrm{Bd} D=F$. Since $D$ is tame, Int $D$ is also tame. By Theorem 3.4 there is a homeomorphism $h: M \leftrightarrow M$ such that $h$ (Int $D)$ is a polyhedron (necessarily infinite) relative to $K(M)$ and $h \mid F$ is the identity. Let $A$ be an annulus in $h(D)$, such that $F$ is a component of $\operatorname{Bd} A$ and the other component of $\mathrm{Bd} A$ is also a polygon. Lemma (2.1) of $\left[\mathbf{M}_{\mathbf{8}}\right]$ asserts that for every neighborhood $U$ of $F$ there is a homeomorphism $h^{\prime}$ : $M \leftrightarrow M$, such that (1) $h^{\prime}(A)$ is a polyhedron and (2) $h^{\prime}$ is the identity at each point of $F$ and at each point of $M-U$. (In fact, this lemma was stated for the case $M=\mathbf{R}^{3}$, but the corresponding result for arbitrary triangulated 3-manifolds follows by the same methods. Since $D$ has a 3-cell neighborhood in $M$, the general result is also derivable from the special result, with the aid of the Hauptvermutung.)

It follows that $F$ is the boundary of a 2-cell which is polyhedral relative to $K(M)$, which was to be proved.

A final remark. In $\$ 1$ it may have seemed more natural to say that a tame 1-sphere is unknotted if it bounds a 2-cell (tame or not). But in fact it makes no difference. Given $J=\operatorname{Bd} D$, where $J$ is tame and $D$ is a 2-cell, let $h$ be a homeomorphism $M \leftrightarrow M$ such that $h(J)$ is a polygon. We can then show that $h(J)$ is the boundary of a polyhedral 2-cell; the proof is a minor variation on the familiar process by which the Dehn Lemma is deduced from the Loop Theorem. (See, for example, [MGT, pp. 197-199].) Therefore $J$ is the boundary of a tame 2-cell, and $J$ is unknotted in the sense defined in $\S 1$.

\section{BIBLIOGRAPHY}

[B1] R. H. Bing and R. J. Bean (Ed.), Topology seminar, Wisconsin, 1965, Ann. of Math. Studies, No. 60, Princeton Univ. Press, Princeton, N. J., 1960, p. 82.

[B $\left.\mathrm{B}_{2}\right]$ R. H. Bing, An alternative proof that 3-manifolds can be triangulated, Ann. of Math. (2) 69 (1959), 37-65.

[B $\left.\mathbf{B}_{3}\right]$, Inequivalent families of periodic homeomorphisms of $E^{3}$, Ann. of Math. (2) 80 (1964), 78-93.

[B 4 ] Armand Borel, Seminar on transformation groups, Ann. of Math. Studies, No. 46, Princeton Univ. Press, Princeton, N. J., 1960.

[B] Glen E. Bredon, Orientation in generalized manifolds and applications to the theory of transformation groups, Michigan Math. J. 7 (1960), 35-64.

[E] C. H. Edwards, Concentricity in 3-manifolds, Trans. Amer. Math. Soc. 113 (1964), 406-423.

[FA] Ralph H. Fox and Emil Artin, Some wild cells and spheres in three-dimensional space, Ann. of Math. (2) 49 (1948), 979-990.

[M] Edwin E. Moise, Periodic homeomorphisms of the 3-sphere, Illinois J. Math. 6 (1962), 206-225.

$\left[\mathrm{M}_{4}\right] \longrightarrow$ Affine structures in 3-manifolds. IV. Piecewise linear approximations of homeomorphisms, Ann. of Math. (2) 55 (1952), 215-222.

[M $\mathbf{M}_{\mathrm{s}}$, V. The triangulation theorem and Hauptvermutung, Ann. of Math. (2) 56 (1952), 96-114. 
$\left[\mathrm{M}_{\mathrm{d}}\right] \longrightarrow$, VIII. Invariance of the knot-types; local tame imbedding, Ann. of Math. (2) 59 (1954), 159-170.

[MGT] __ Geometric topology in dimensions 2 and 3, Springer-Verlag, New York, 1977.

[P] C. D. Papakyriakopoulos, On solid tori, Proc. London Math. Soc. (3) 7 (1957), 248-260.

[S] P. A. Smith, Transformations of finite period. II, Ann. of Math. (2) 40 (1939), 690-711.

[S $]$ _ Periodic transformations of 3-manifolds, Illinois J. Math. 9 (1965), 343-348.

[St] John Stallings, On the loop theorem, Ann. of Math. (2) 72 (1960), 12-19.

Department of Mathematics, Queens College, (CUNY), Flushing, New York 11367 